UNIVERSITE DU QUEBEC A CHICOUTIMI

\author{
MEMOIRE DE RECHERCHE \\ ETUDES RN MATTRISE REgGIONALES
}

PAR

PATRICE FORTIN

L' IMPLICATION REGIONALE DES MEDIA ECRITS

DANS LES GRANDS DEBATS POLITIQUES CANADIENS

LE 23 JUIN 1988 


\section{Bibliothèque}

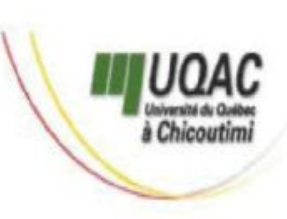

\section{Mise en garde/Advice}

Afin de rendre accessible au plus Motivated by a desire to make the grand nombre le résultat des results of its graduate students' travaux de recherche menés par ses research accessible to all, and in étudiants gradués et dans l'esprit des accordance with the rules règles qui régissent le dépôt et la governing the acceptation and diffusion des mémoires et thèses diffusion of dissertations and produits dans cette Institution, theses in this Institution, the I'Université du Québec à Université du Québec à Chicoutimi (UQAC) est fière de Chicoutimi (UQAC) is proud to rendre accessible une version make a complete version of this complète et gratuite de cette œuvre. work available at no cost to the reader.

L'auteur conserve néanmoins la The author retains ownership of the propriété du droit d'auteur qui copyright of this dissertation or protège ce mémoire ou cette thèse. thesis. Neither the dissertation or Ni le mémoire ou la thèse ni des thesis, nor substantial extracts from extraits substantiels de ceux-ci ne it, may be printed or otherwise peuvent être imprimés ou autrement reproduced without the author's reproduits sans son autorisation. permission. 


\section{RESUMR}

La présente étude s'inscrit parmi celles qui traitent des média écrits du Saguenay-Lac Salnt-Jean au XXème siècle. Donc la matière brute de notre travall aura une essence qui se voudra réglonale. C'est un point qu'il ne faut pas perdre de vue. De plus notre mémoire est basé sur quatre grands débats politiques canadiens qui ont touché le SaguenayLac Saint-Jean. Évidemment ce sont des événements quí ont aussi secoué le Canada et le reste du Québec. Telle est la dynamique à explorer. Ces débats politiques sont: La conscription de 1917, le plébiscite de 1942, la mort de Pierre Laporte $(1970)$ et le référendum de 1980 . Des événements qui suscitent encore d'allleurs aujourd'hui la controverse. Chacun de ces débats politiques aura un chapitre dans notre thèse. Nous définirons $1 \mathrm{e}$ contexte général de 1 'époque et plus particulièrement celul qui régnait dans notre région. Chaque chapitre aura une analyse de contenu des journaux locaux qui sera limitée mais pertinente. Car n'oublions pas qu'une analyse de contenu complète de chacun de ces débats pourrait entrainer à elle seule une thèse de maltrise. Nous nous servirons de sources Imprimées comme: Le Progrès du Saguenay et L'Étoile du Lac pour la période 1917-1918. Ce sera 1a même chose pour 1'époque 1942-1943. E1les seront différentes pour la mort de Pierre Laporte puisque nos sources imprimées seront: L'Étolle du Lac (1970), le Progrès-Dimanche (1970) et Le Soleil (1970). Flnalement pour le référendum de 1980 nous utiliserons: L'Étolle du Lac et Le Progrès-Dimanche de 1980.

Des études générales, des articles et des études de propagande complèteront notre analyse. Ces analyses de contenu démontreront que le Saguenay Lac Saint-Jean $n$ 'a jamais souffert d'une pénurie d'information politique quelconque. Cette situation n'a pas existé au XXème siècle. Le journal a bien rempli sa mission. Le décodage des messages des média écrits le montre clairement. Le discours analysé respecte généralement la vérité. L'opinique publique n'a pas été trompeee. C'est une réalité à ne pas oublier. Les journaux francophones du Saguenay n'ont pas "exagéré" les événements décrits. Avec le recul qui s'impose nous pouvons 1 'affirmer. Les données quantitatives transformées en variables le prouvent assurément.

Les média écrits du Saguenay ont été continuellement hantés par les conflits politiques qui ont secoué dans ce siècle le Canada et le Québec. Les média écrits du Saguenay Lac Saint-Jean n'ont jamais été des espaces fermés. Le décalage au niveau des grands débats politiques canadiens n'existe pas. La presse écrite réglonale n'est pas exempte des consultations publiques qui ont fait la manchette au Québec. Le domaine des mass média écrites ne souffre pas d'une "rupture" avec le reste du pays. La population du Saguenay ne fut pas laissée dans 1'ignorance. Notre travail le prouve raisonnablement. Le Saguenay a suivi 1'évolution du reste du Québec. La dynamique historique est la même dans les deux cas. 
Un exemple, le rôle du clergé a changé fondamentalement. Si on regarde dans les grandes lignes, notre traval1, on notera un déclin sensible et visible. En 1942, alors que 1'influence de 1'Église catholique était à son sommet, le clergé parle de concert avec le consell central des Syndicats nationaux catholiques du diocèse de Chicoutimi. On ne retrouvera plus une telle manifestation dorénavant. En 1970, évidemment le clergé condamnera durement $I^{\prime}$ exécution de Plerre Laporte. Il n'est plus aussi puissant que le pouvoir civil cependant. L'autorité du clergé est désormals spirituelle et morale. Son pouvoir s'arrête là. Finalement, en 1980 , le clergé refuse tout simplement de prendre position officiellement dans les média écrits. La révolution tranquille a changé bien des choses et cela même à un niveau régional.

Dans le Québec moderne, ce sont les politiciens et les autorités civiles qui accaparent 1 'attention des média écrits. Ils détiennent le monopole de la parole écrite. Cette situation se reflète au Saguenay. Notre région a été toujours un bastion du fait français en Amérique. Par exemple, lors des débats publiques de 1917 et 1942, les profondes contradictions qui existalent entre le Canada anglais et le Canada français se retrouvent précisément dans la presse du Saguenay. Le meurtre de Plerre Laporte fut condamné par les journaux francophones du Québec. On retrouve cette condamnation unanime dans les média régionaux pendant la crise d'Octobre. Encore une fois, le Saguenay n'est pas en marge du reste du Québec. Les journaux régionaux ont donc eu des positions comparables à celles adoptées par les Imprimés de Québec et de Montréal. 
REMMRCIBMENTS

La réalisation de ce travall a nécessité la collaboration de plusieurs personnes. Aussi, je tiens à les remercler pour 1'alde apportée.

Je pense d'abord a M. Paul Pllisi et M. Jean-Guy Genest qui m'ont guidé tout au long de 1 a recherche et de la rédaction et dont j'ai utilisé grandement leur expertise. Je pense aux professeurs M. Gilbert Larochelle de 1'U.Q.A.C. et à M. Richard Jones de I'Université Laval. Je pense aussl aux personnels de la Blbllothèque de 1'U.Q.A.C. et du dêpôt régional des archives nationales du Québec à Chicoutimi qui m'ont beaucoup aidé dans mes recherches.

grand merc1.

A toutes ces personnes, et à toules celles que f'oublie, un 


\title{
LISTB DES TABLEAUX
}

\begin{abstract}
page
TABLEAUX sur la conscription de 1917 . . . . . . 30 a 37 et 42

TABLEAUX sur le plébiscite de 1942 . . . . . . 54 à 61 et 65

TABLEAUX sur la mort de Plerre Laporte . . . . . . 77 a 84 et 90
\end{abstract}

TABLEAUX sur le référendum de 1980 . . . . . . 102 à 109 et 115 


\section{INTRODUCTION}

Notre thèse a pour titre: "L'implication régionale des média écrits dans les grands débats politiques canadiens". Notre mémoire est basé sur quatre grands débats politiques quí ont touché le Saguenay-Lac Saint-Jean. Évidemment, ce sont des événements quil ont aussi touché le Canada et le reste du Québec. Telle est la dynamique à explorer. Notre méthodologie consiste essentiellement à la compilation quantitative de la perception d'éléments par des média régionaux. On pourrait nous reprocher qu'11 s'aglt d'une technique sommalre plutôt qu'une méthode proprement dite. A cet argument, nous répondrons que les techniques d'analyse sont valables dans la mesure où le chercheur se limite à la stricte perception des événements par les média régionaux écrits. C'est ce que nous faisons justement. Cependant, 11 nous faut rappeler au lecteur de cette thèse que les média écrits ont toujours influencé une part plus ou molns importante de la population.

Le Saguenay Lac Saint-Jean est situé approximativement à 200 kilomètres de Québec. I1 contient près de 300000 habitants. Son act1vité économique repose sur 1'exploitation de ses trois grandes resssources naturelles qui sont la terre (agriculture et mines), l'eau (électri- 
cité et vole maritime) et le bois. Nous ne donnerons pas une définition du milieu régional saguenéen car ce n'est pas l'objet du présent travail. Notre tdée est de rassembler en une étude comparative quatre grands épisodes de 1'histoire du Québec et de mesurer leurs incidences dans les média écrits du Saguenay-Lac Saint-Jean.

Nous voulons faire un rapprochement entre des événements-charnlères importants dans la presse écrite et donner à notre travail une dimension nouvelle dans un cadre comparatif. Alnsi, nous apporterons un éclalrage Inédit sur 1'évolution de la presse au Saguenay. Notre cadre théorique est constitué en fait d'une technique d'observation du matériau journalistique dont le déploiement sera au service d'une interprétation d'ensemble au niveau des média écrits.

Nous ne parlons pas de crise politique mais de débat ou de consultation publique majeure. Une série de débats populaires a conduit à "1a conscription de 1917". Dans ce cas, on pourrait même parler de crise civile à la suite des émeutes de Québec. "La conscription de 1942" n'offre certainement pas une situation pareille. Tout est resté dans un cadre constitutionne1. "Les événements d'octobre de 1970" ressemblent 
au premier cas étudié. Le référendum de 1980 fut une consultation populaire pacifique sans germe d'une révolte quelconque. C'est pour ces raisons que nous prenons le terme "grands débats politiques". Chacun de ces spasmes politiques constituera un chapitre dans notre travail. Chaque chapitre aura une analyse de contenu de journaux locaux qui sera limitée mals pertinente. Nous nous servirons des sources imprimées comme Le Progrès du Saguenay et L'Étolle du Lac pour la période de 1917-18. Ce sera le même procédé pour 1'époque 1942-1943.

Notre travali de compliation des récurrences quantitatives des titres de journaux évoque des attitudes politiques. Elles ne sont pas toujours révélatrices de la situation de 1'ensemble du Saguenay. Elles peuvent être à la mesure des oplnions du journaliste signataire des articles dépouillés ou encore d'un organe de presse en particulier. Les média effectuent plutôt un découpage de la réalité soclale. En tous les cas ce sont les théories contemporaines de l'information et des communications qui le disent. Ce serait faire trop confiance à la représentativité politique des média que de les percevoir comme un reflet parfalt de 1a population à laquelle ils s'adressent. 
La récurrence des titres de journaux ne pourraft mesurer que 1'opinion des élites de l'époque ou de tel ou tel groupe situé en position dominante de pouvoir au sein d'une organisation de presse. Donc, 11 n'y auraft pas nécessairement équivalence entre le discours de 1'imprimé et ce qu'une population tient pour vérité.

Nos sources écrites pour les mesures de guerre d'Octobre 1970 seront Le Progrès-Dimanche, Le Sole11 et L'Étolle du Lac. Finalement, pour le référendum de 1980, nous utiliserons L'Étoile du Lac et Le Progrès-Dimanche. Des études générales, des articles et des études de propagande complèteront notre analyse. L'analyse des média écrits nous montre généralement des aspects inconnus de notre société. Il n'est pas question de refaire 1 'histolre de ces grandes questions. D'ailleurs, ces histoires ont déjà été faites. Nous allons donc réduire sensiblement I'espace que nous consacrons au contexte général pour mettre 1'accent sur ce quil est original dans notre thèse, c'est-à-dire le comportement des média écrits du Saguenay-Lac Saint-Jean. C'est le défi que nous avons relevé.

\footnotetext{
Certaines valeurs qui existent dans une société peuvent très bien se reflêter dans des commentaires écrits et cela d'une manière
} 
très subtile ou même détournée. Pour faire une telle analyse, on dolt se servir de données quantitatives qui se transformeront en variables. Moeurs et normes demeurent des mécanismes de régulation sociale. Les détenteurs du discours peuvent nous révéler des faits apparemment évidents mais qui restaient obscurs. Il s'agit d'identifier un groupe, une catégorie ou une classe soclale par ce procédé. Par exemple, étaient-ce les religieux qui prenalent la parole lors des premiers débats analysés? Après la révolution tranquille, étalent-ce au contraire les politiciens et les autorités civiles qui détenaient le monopole de la parole écrite?

Notre thèse démontrera les profondes contradictions qui existent entre le Canada anglais et le Canada français. On retrouve d'a11leurs ces mêmes contradictions dans la presse écrite au Saguenay. On a parlé de falre un référendum en 1917. Une pareille dynamique s'est répercutée jusqu'à une région comme la nôtre. A un certain niveau, on peut parler de vote ethnique et même de "guerre civile verbale". La conscription de 1942 est la continuation de celle de 1917. Le cardinal Villeneuve, Archevêque de Québec, a pris parti pour la politique de guerre du gouvernement King. Le bas clergé a-t-11 adopté 1 a même position au Saguenay-Lac Saint-Jean dans les journaux de l'époque? 
La crise d'Octobre est l'aboutissement d'un plus long processus. On parle alors de l'activité "illégale" d'un groupe armé, lequel vise une révolution nationale en vue d'établir 1'indépendance. Les origines de ce mouvement remontent aux années soixante. Un groupe terroriste cherche 1a destabilisation de l'État fédéral. C'est une première. C'est une crise qui grandira, et qui trouvera son apogée dans les mesures de guerre. L'entrée en scène du Partí québécols a joué aussi un rôle. On sait que les perquisitions et les arrestations ont frappé toutes les régions du Québec. Militants syndicaux, groupes populaires, comités de citoyens, mouvements étudiants, associations nationalistes et organisations politiques de gauche furent les principales cibles de la police. Notre région n'a pas été l'exception qui confirme la règle.

Nous analyserons finalement le référendum de 1980 dans la presse régionale. Cette fols-ci le Parti québécois dans le cadre du référendum favorise une évolution constitutionnelle 1égale. Le débat est majeur et 11 se résume à deux positions: on est pour ou contre la souverainetéassoctation. En général, les journaux francophones sont restés neutres "officiellement". La presse anglophone demeurait résolument pour le non. Curieusement notre région a voté massivement pour le oui. C'est d'ail- 
leurs 1'endrolt où le oui a été le plus populaire. Était-ce son élolgnement des grands centres qui a été la cause de cette situation? Ou étaitce le petit nombre d'anglophones résidant au Saguenay quí fut le facteur déterminant dans le vote? Ce sont des éléments à prendre en considération.

Dans les quatre périodes qui nous intéressent, nous devrons emprunter un certain nombre d'expressions linguistiques afin de donner un tableau plus juste des époques analysées. Ces emprunts peuvent varier d'un chapttre à 1'autre car 1a dynamique historique change constamment. On ne parle pas "du mur de Berlin" en 1917. On en parle dans les années 60 cependant. De plus, certaines expressions sont significatives seulement si elles sont employées dans un contexte québécois.

Nous n'avons qu'une seule et unique hypothèse. Les média écrits du Saguenay-Lac Salnt-Jean (et cela même s'ils sont éloignés des grands centres) ne sont pas des vases clos. Il n'y a pas eu décalage au niveau des grands débats politiques canadiens. La presse écrite n'est pas exempte des bouleversements qui ont secoué 1e reste du Québec. I1 
n'y a jamais eu de pénurie d'information écrite au Saguenay-Lac SaintJean. C'est d'allleurs pour cette raison qu'on y retrouve les profondes contradictions qui ont bouleversé le Québec et le Canada. On ne peut pas parler au Saguenay de "rupture" avec le reste du pays dans le domaine des mass média écrites. La population du Saguenay ne fut pas laissée hors des grands débats politiques canadiens. Ce sont d'ailleurs les mêmes é1ites qui demeurent les détenteurs du discours écrit dans les journaux régionaux. Cette situation a duré jusqu'à la révolution tranquille. Ainsi le clergé aura une part importante du discours écrit jusqu'au début des années 60. Ensulte, les autorités civiles (policiers, politiciens, notables, etc.) prendront la relève. Une évolution sensiblement pareille à celle qu'a subie l'ensemble de la soclété québécoise. Ce facteur tendrait à prouver que le Saguenay,n'est qu'une extension d'un ensemble plus global.

Le facteur intéressant, c'est de faire un parallèle entre les conflits qui hantalent le Canada et le Québec d'une part, et notre univers régional, d'autre part. La rumeur populaire assurait que le Saguenay formait un ensemble à part. L'éloignement en était la cause. Plus récemment, Claude Ryan 1'a répété après le référendum de 1980 . Nous, nous considérons au contraire que ce facteur n'est pas valable au niveau des communications écrites. 
On salt que le Saguenay possède une des plus fortes majorités de francophones de tout le Québec, plus de $90 \%$. C'est un facteur à ne jamais oublier. C'est ce qui expliquerait, selon nous, les positions nationalistes des journaux. Donc 1'élément culture1 compte beaucoup dans notre thèse. Les prises de position des journaux locaux ne différeront pas fondamentalement des écrits dits "nationaux". I1 n'existe pas un milieu local, un milieu provincial et un milieu national. A 1'inverse, les trois milieux forment un ensemble intimement lié. C'est d'ailleurs là que réside la grande difficulté d'un tel travail. Il faudra diviser et justifier exactement chacune de ses parties.

Le décodage du message écrit peut prendre plusieurs formes. On peut déterminer la quantité de ce message en variables. Dans les analyses de journaux, 11 faut cependant spécifier si ce sont des éditoriaux, des lettres des lecteurs ou des descriptions d'événements. De plus, il faut indiquer la provenance et 1'identité des émetteurs cités.

Ensulte, on passe à la rédaction des catégories. C'est à ce moment que le chercheur doit faire particulièrement attention. En effet, 
par exemple, trouvez-vous un synonyme d'Incendie découlant d'un acte prémédité. Donc on doit agir par déduction: combien de fois retrouvera-ton le mot incendle à la place de sabotage ou de feu criminel? Combien de fois retrouvera-t-on 1'inverse de la formulation? C'est une analyse qui se voudra objective (mêmes résultats), systématique (catégories chotsies), quantitative (leur fréquence) et dirigée finalement. A ce niveau le quantitatif compte beaucoup. Cependant, n'oublions pas le qualitatif, une phrase comme: "C'est un fil électrique qui a mis le feu", peut être très significative dans un certain contexte, si on recherche la trace d'une rumeur lancée par les journaux.

De plus, on doit noter le nombre d'articles dans le cas qui nous intéresse. Nous compterons la fréquence du mot et non pas le nombre de lignes ou de centimètres qu'il occupera. Certalns événements peuvent avoir une place de premier plan dans les journaux régionaux (haut de la lère page) et une position assez secondaire, chez les périodiques métropolitains (bas de la lère page et même 3 ou 4lème page). Ce fut le cas pour 1'incendie de 1'hôpital de Chicoutimi. Le grand avantage d'une telle étude demeure le fait qu'il n'y a pas d'éléments linguistiques qui interfèrent entre les rédacteurs du discours (les journalistes et son lecteur (nous)). En effet, $11 n^{\prime} y$ a pas de rédacteur professionnel comme pour un homme politique. De plus, le problème de traduction ne se pose pas non plus. 
Pour analyser la rumeur suggérant la participation du F.L.Q. a l'Incendie de 1'hôpital de Chicoutimi, nous avions établi cinq grandes catégories pour cerner le message: "vocabulalre relevant d'un accident naturel, manffestation d'autorité, représentants des forces de 1'ordre, vocabulaire relevant d'une agression collective et finalement manifestation du terrorisme". C'est un type semblable de grille d'analyse qui nous servira dans notre travail final. On trouve aux pages 12 et 13 la forme que prendront ces grilles d'analyse. 


\section{TABLEAU I}

\section{CATÉGORIBS}

VOCABULAIRE RELEVANT D'UN ACCIDENT NATUREL MANIFESTATION D'AUTORITE

REPRESENTANTS DES FORCES DE L'ORDRE

VOCABULAIRE RELEVANT D'UNE AGRESSION COLLECTIVE

MANIFESTATION DU TERRORISME 
12 Vocabulaire relevant d'un accident naturel

8 Manifestation d'autorité

3 Représentants des forces de 1'ordre

6 Vocabulaire relevant d'une agression collective

3 Manifestation du terrorisme

93 Vocabulaire relevant d'un accldent nature1

23 Manifestation d'autorité

27 Représentants des forces de 1'ordre

22 Vocabulaire relevant d'une agression collective

12 Manifestation du terrorisme

2 Vocabulaire relevant d'un accident naturel

1 Manifestation d'autorité

2 Représentants des forces de 1'ordre

1 Vocabulaire relevant d'une agression collective

0 Manifestation du terrorisme

2 Vocabulaire relevant d'un accident naturel

o Manifestation d'autorité

0 Représentants des forces de 1'ordre

4 Vocabulaire relevant d'une agression collective

0 Manifestation du terrorisme 
Ce sont ces tableaux qui nous indiqueront la provenance des catégories et leur fréquence. A la lumière d'une telle analyse, nous pouvons affirmer que l'événement analysé occupait une place de choix dans les quotidiens régionaux (Progrès du Saguenay et Le Sole11) et une place molns enviable dans les journaux provinclaux (La Presse et le Devoir). D'ailleurs le nombre des fréquences des catégories 1'indique assez clairement. On fait mention dix fols plus de l'événement analysé dans nos productions réglonales. C'est une situation au fond parfaitement norma1e. Un fait marquant à Chicoutimi ne 1'est pas nécessairement à Montréal. On trouve dans les cinq pages suivantes les tableaux produits en fonction des catégorles cholsies.

Mais en général, nous savons que les grandes révolutions se sont faltes à partir des grands centres urbains. Nous considérons que les changements sociaux et politiques peuvent suivre le même chemin. Les média écrits ne feraient pas exception à la règle. Le centre communique ses réactions a la périphérie. Ce n'est pas le contraire qui s'est passé au Québec. Par exemple, une action du F.L.Q. a Québec ou à Montréal frappait l'imagination populaire. A ce niveau, la couverture des média importait beaucoup. Comparativement aux grands centres, notre région comptait un pourcentage beaucoup plus élevé de cellules "terroristes". 
TABLEAUX III

VOCABULAIRB RELEVANT D'UN ACCIDENT NATUREL

MOTS

FREQUENCES

Incendies \& incendie

Feu \& feux

Sinistre (1e)

4

Flammes

10

Êlément destructeur

Catastrophe

Conflagration

Débris fumants

Départements dévastés

Filet de fumée

Proportions gigantesques

Étincelles

Désastre

Ruines fumantes

1

TOTAL 
Contrôle strict

Service d'ordre

Laissez-passer

Visites suspendues

Surveillance

Étroite survelilance

Arrestation

Faction

Polnts stratégiques

Personne non autorisée

Enquête

Recherches

Témoing

Interroger

Garde

Contrôle

Investigation (s)

TOTAL 


\section{TABLEAD V}

\section{REPRÉSERTANTS DES FORCES DE L'ORDRE}

MOTS

FREQUENCES

Force constabulatre

Régiment du Saguenay

Agents de la sûreté

8

Sûreté municipale

Armée

Enquêteurs

Procureur

4

Investigateur

1

Source officielle

1

Premier ministre

1

Gouvernement

1

TOTAL 


\section{TABLBAU VI}

\section{VOCABULAIRE RELEVANT D'UNB AGRESSION COLLECTIVE}

MOTS

Menaces

6

Suspects

1

Gestapo

1

Mur de Berlin

1

Désordre

1

Mystẻre

2

Actes de vandalisme

1

Ind Ividus

1

Lettres de menaces

1

Thêâtre de guerre

1

Vanda11smes

2

Instinct de destruction

1

Saccage

1

Main criminelle

4

Intentionne1lement

3

Appe1s anonymes

2

Mettrait le feu 
Quelque chose à volr

$F \cdot L \cdot Q \cdot$

Groupe suspecté

Incendiaire

Cocktail

Découverte d'un détonateur

Cause

Colis
1

1

1

6

2

1

1

2

15
TOTAL

\section{5}

2

2

2

2


CHAPITRE 1

LA PREMIĖRE CONSCRIPTION (1917) DANS

LES MEDIA ECRITS DU SAGUENAY 
En 1910, le premier ministre du Canada, Sir Wilfrid Laurier, présentait un projet de loi visant à créer une marine canadienne qui apporterait sa contribution au renforcement de la flotte impériale britannique. Ce fut le début d'un long débat qui allait déboucher sur des événements dramatiques et cela jusqu'en 1918. Il y a eu a la suite de cette 101 un tollé de protestations de part et d'autre. Mals l'affaire n'allait pas en rester 1a. Selon les nationalistes canadiens-français dirigés par Henri Bourassa, une telle action ne pouvait qu'entraîner tôt ou tard le Canada dans les guerres anglaises. Cette situation servira le conservateur Robert Borden qui, grâce à l'appui des nationalistes français remportera les élections.

Sir Robert Borden remerciera d'allleurs ses nouveaux alliés. "When the new Conservative government took power, Sir Robert Borden naturally offered places in the cabinet to the Nationalist allies who had been so instrumental in reducing the Liberal majority in Quebec".(1) Donc en 1911, le Canada était dirigé par le conservateur Robert Borden. Le Canada avalt une armée de métier de 3000 hommes et une milice de 35000

1 - Elizabeth Howard Armstrong, The Crisls of Quebec, 1914-1918, Toronto, McC1e11and and Stewart, $1937, \mathrm{p} .32$ 
volontaires. Cette armée était majoritairement anglophone. En 1912, Borden proposait le don d'une somme de 35 millions de dollars à la marine anglaise.

Au fond, que ce soit un conservateur ou un libéral au pouvoir ne changeait rien a la situation. L'entrée en guerre de la métropole anglaise signifiait un état de belligérance pour ses colonies. Le Canada en faisalt partie. Ce n'était donc plus qu'une question de temps. Les Canadiens français n'avaient guère un sentiment profond ni pour 1'Angleterre, ni pour la France à cette époque. I1s possédaient cependant de 1'amitlé pour cette dernière. Pour eux, leurs devoirs étaient plutôt de protéger les minorités françaises des autres provinces. "Ils s'intéressaient aux droits des Franco-canadiens partout au Canada et 11 s protestaient contre chacune des tentatives faites pour les en priver".(2)

Justement en 1912, 1'Association canadienne-française d'éducation d'Ontario commençait une lutte pour la défense des droits scolalres

2 - Robert Choquette, Langue et religion Histolre des conflits ang1ofrançals en Ontario, Ottawa, les Editions de I'Université d'ottawa, $1980, \mathrm{p} .115$ 
de la minorité française de cette province. Ce conflit devalt prendre une ampleur inégalée. I1 allait diviser profondément la nation à un moment critique. Le débat devalt prendre une place considérable sur la scène publique et on créalt une commission d'enquête, 1a commission Merchant. Elle slégea pendant plusieurs mols avant de rendre son rapport au gouvernement ontarien.

L'Association d'éducation franco-ontarienne décida de résister. Une longue batallle juridique devait ainsi commencer. Le malaise causé sera considérable et profond. Les Franco-Ontariens fondèrent même un journal pour se défendre: le premier numéro du Drolt parut le 27 mars 1913. Un réseau de presse fut ainsi bientôt créé L'Action Sociale de Québec, Le Devoir de Montréal, Le Drolt d'ottawa, La Liberté de Winnipeg et Le Patriote de Régina.

A partir de ce moment tout était en place pour que le conflit prenne une envergure nationale dans une conjoncture qu'on peut qualifier de critique. Le Canada lui-même dans une guerre mondiale allatt vivre un 
conflit intérieur majeur. "Au moment où le Québec entrait dans 1'année 1915, son attention et sa sympathie étalent beaucoup plus concentrées sur 1'Ontario que sur 1'Europe".(3) Le "malalse" allalt se répercuter jusqu'au Saguenay. Le 4 août 1914, c'est la déclaration de guerre de la Grande-Bretagne. Le gouvernement canadien vote un crédit de 50 millions de dollars pour la formation d'une armée canadienne. C'est la renaissance du débat.

Pourtant, au début 1e consensus étalt fermement établi. L'effort de guerre semblait aller bien superficlellement. A cet instant la puissance militaire du Canada est quasi inexistante. Par exemple, 1a marine canadienne demeuralt encore a l'état de projet. La puissance militalre canadienne pouvait tout au plus assurer la sécurité intérleure du pays. E1le ne pouvait certes pas affronter un adversaire puissamment armé ou même moyennement. Néanmoins, la presse sera unanime dans son appul au gouvernement d'envoyer un contingent de 20000 hommes au secours de la mère-patrie. C'étalt le 6 août 1914. Cependant tout le monde pensalt que la guerre durerait peu longtemps.

3 - Mason Wade, Les canadiens françals de 1760 a nos jours, Ottawa, Le Cercle du Livre de France, 1963, T.II, p.65 
La campagne de recrutement allait bien: a la fin de juin 1915, 100000 hommes s'étalent portés volontaires et au terme de la même année, on arrivalt au nombre de 217000 . Ce sera la presse anglophone, conservatrice et libérale, qui demandera au gouvernement d'augmenter son effort de guerre par une participation accrue au front. Les organisations de recrutement étaient majoritairement anglaises. Ce seront d'ailleurs, elles, qui exigeront les premières la conscription. Le Québec allait devenir de plus en plus lsolé et divisé. "But for Québec the Great War produced only bitterness and division".(4) De plus, le françals en Ontario n'arrangeait rien.

L'année 1916 fut néanmolns celle de l'enrôlement. Cependant la contestation grandissait. En effet, le conflit ontarien étalt à son apogée. La bataille juridique dépassait les frontières de 1'Ontario. Le débat fut soulevé devant le Parlement. Tout cela n'allait certes pas aider la cause du recrutement au Québec. Et c'est finalement à partir de décembre 1916 que les chiffres des volontaires vont balsser dramatiquement. Borden ne peut plus maintenir les engagements militalres canadiens sans la participation du Québec.

4 - J. L. Granatstein et J. M. Hitsman, Broken Promises: A History of Conscription in Canada, Toronto, Oxford University Press, 1977, p.98 
Durant 1'hiver 1916-1917, les associations de recrutement ont été pratiquement inutiles. A ce moment, les journaux canadiens-français sont contre l'idée d'Intensifier le recrutement. Seule la presse est pour mais dans la perspective d'éviter la conscription. La participation des élites canadiennes-françaises a été pratiquement inexistante dans 1a campagne de recrutement. Le clergé, les hommes publics, le gouvernement provincial et les chefs locaux n'embarquent pas dans le processus. La presse anglophone réclame la fermeture du Devolr. Mals Borden a peur de la réaction du Québec. Il a même peur d'une guerre civile au Québec à 1a suite de 1a conscription. Le 29 mai 1917, Borden proposera a la Chambre des communes la conscription. E1le est approuvée le 24 juillet par 162 volx contre 44 .

Le Québec allait vivre des jours sombres. Pendant tout 1'été 1917, il y avait eu des manifestations hostiles à la conscription. Les solrs du 29 et 30 août, 11 y eut un tué et quatre policlers blessés. Le 9 août la propriété de Lord Atholstan (Hugh Graham) à Cartierville, dont 1e Montreal Star soutenait activement la conscription, a été carrément dynamitée. Un certaln Élie Lalumière et onze autres citoyens furent arrêtés. Lalumière avoua des complots pour détruire le Star, le Mount Royal Club et pour tuer le premier ministre Borden. 
Le lundi de Paques, le ler avril 1918, à Québec une émeute éclate. Les résultats seront dramatiques: quatre tués, 40 blessés et soixante-deux arrestations. A partir de ce moment, la ville sera sur un pied de guerre.

Analyse de contenu des journaux locaux

Notre analyse de contenu porte sur les émeutes de Québec. SeIon la manière exposée dans l'introduction, nous décoderons le message proposé par les journaux régionaux et le quantifierons en variables. Le premier hebdomadaire analysé sera "Le Progrès du Saguenay". Ce journal est né quand le Saguenay ne possédalt qu'une population de 20000 personnes et une seule ville. Il fut selon les époques hebdomadaire, bi-hebdomadaire et même quotidien. Au moment qui nous intéresse plus particulièrement c'est un hebdomadaire. En 1918, chaque page a cinq colonnes et

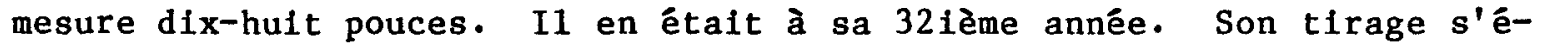
levait à 4550 coples approximativement. Le deuxième hebdomadaire analysé sera: L'Étolle du Lac appelé à cette période Le Colon du Lac SaintJean. Ces renselgnements proviennent de 1'ouvrage sur la presse au Saguenay, de Raoul Lapolnte, Histolre de 1'Imprimerie au Saguenay (18791969). 
Dans 1'échantillon analysé, 11 n'y a pas eu d'éditoriaux, ni de lettres au journal qui parlaient de l'évévement cité. Les émetteurs analysés furent donc: Le Progrès du Saguenay et Le Colon. Le récepteur fut, dans son immense majorité, 1'homme de la rue de 1a région. Au fond, 1a grande question que nous posons est la sulvante: comment ont été perçues les émeutes de Québec par les médlas écrits du Saguenay-Lac SaintJean? Comme nous l'avons dit, 1a grande difficulté d'un tel travall reste la classification des catégories. Donc, nous agirons par déduction. C'est une analyse objective (mêmes résultats), systématique (catégories) et quantitative (leur fréquence) que nous proposons.

Nous avons pris comme échantillon la totalité des documents portant sur les émeutes de Québec. C'est le quantitatif (1a fréquence) qui compte surtout dans ce type de trava11. Les journaux régionaux n'ont rien fait de véritablement significatif pour envenimer le climat de violence qui existait déjà au Québec.

I1 ne faut pas simplement comptabiliser tous les mots sans tenir compte non seulement du contexte particulier où ils sont placés, mais aussi dans leur définition plus large. On doit falre constamment 
des emprunts à la 1inguistique. Notre étude porte sur 13 articles d'information exclusivement. Notre travall couvre une période de deux mois (avril et ma1).

Nous avons compté le nombre de fols que revenait le mot et non pas le nombre de 1ignes ou de centimètres qu't1 occupalt. On peut dire que l'événement analysé a occupé une place consldérable dans les journaux réglonaux (toujours au haut de la première page). Le Colon et Le Progrès du Saguenay ont 13 articles quil parlent des émeutes de Québec. Nous avons privilégié les articles portant sur les émeutes de Québec à cause de 1'ampleur de la couverture de cet événement dans 1a presse régionale, et du fait aussi qu'on jugeait la politique du gouvernement fédéral par la même occasion. On trouve dans les huit pages suivantes les tableaux établissant les catégories cholsies ainsi que leur fréquence par journal. 
TABLEAU I

\section{CATÉcories}

VOCABULAIRE DESAPPROUVANT LA POLITIQUE DU GOUVERNEMENT FÉDÉRAL

MANIFESTATION D'AUTORITÉ

REPRESENTANTS DES FORCES DE L'ORDRE

VOCABULAIRE RELEVANT D'UNE AGRESSION COLLECTIVE

ENCOURAGEMENTS A LA VIOLENCE 
20 Vocabulałre désapprouvant 1a polltique du gouvernement fédéral

1 Manifestation d'autorité

4 Représentants des forces de 1'ordre

14 Vocabulaire relevant d'une agression collective

0 Encouragements a la violence

24 Vocabulaire désapprouvant la politique du gouvernement fédéral

47 Manifestations d'autorité

57 Représentants des forces de 1'ordre

30 Vocabulaire relevant d'une agression collective

0 Encouragements à la violence 
TABLEAU III

VOCABULAIRE DESAPPROUVANT LA POLITIQUE DU GOUVERIEMENT FEDERAL

MOTS

FREQUENCES

Fausse manoeuvre

Application même arbitraire

Intempestive 2

Lol adoptée malgré nous 2

Contre nous 2

Griefs 2

Subissons 2

Abus d'autorité 2

Poussés malgré eux 2

Toutes sortes de vexations 2

Provoqués par de sales agents bien payés 2

Leurs sinistres besognes 2

Lutter dignement $\quad 2$

Irréligion 2

Fanatisme 2

Patriotisme $\quad 2$

Évitons le désordre 2

Marchons toujours dans le chemin de 1 'honneur 2

Profané 1'exercice du droit de suffrage 2

\section{1}

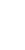
2 2 2

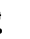

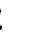
2 2 
TABLEAU III

VOCABULAIRE DESAPPROUVANT LA POLITIQUE DU GOUVRRNEMENT FEDERAL

SUITE

MOTS

FREQUENCES

Traités brisés

2

Institutions ébranlées

2

Procédé 1gnoble

2

TOTAL

44 


\section{TABLEAU IV}

\section{MANIFESTATION D' AUTORITE}

Lo

Manière forte

2

Loi martiale

Suppression

2

Événements

2

L'ordre

2

Loi mflitaire

2

L'enrôlement

2

L'autorité locale

2

Opérations militaires

A la force

2

Contrôle

2

Loi fédérale

2

Force militaire

2

Arrestation

2

Conscrit

2 
Soldats

Gouvernants, Députés et Politiciens

Satellites

Législateurs

Parlement

Colone1

Premier ministre

Ministres

Régiment et régiments

Chambre

Militaire et militalres

Milice

Gouvernement

Troupes

Patrouf1le 
Evénements

2

Gravité exceptionnelle

Regrettable

1

Pour ne pas dire plus

1

Conséquences fâcheuses

1

Race canadienne-française

2

Bien mauvais pas

1

Spectateurs horrifiés

1

Désordres

1

Élément canadien-français

1

Survivance de sa fol

1

Survivance de sa langue

1

Manifestation, manifestants

12

Émeutes, émeutiers, émeutes de Québec

Blessés

Foule énorme

Guerre 
TABLEAU VII

ENCOURAGEMENTS A LA VIOLENCE

MOTS

FRÉQUENCES

Aucun

0

TOTAL 
Résultats de l'analyse de contenu

La catégorle: "encouragements à la violence" ne recueille aucune fréquence. On ne trouve pas non plus de phrases comme: "Luttons mes frères ou résistons par les armes". Cette situation est particulièrement significative de la conjoncture politique existante au SaguenayLac Saint-Jean à cette époque. Evidemment, la presse régionale est contre la politique fédérale. Le nombre de fréquences de la catégorie: "vocabulaire désapprouvant la politique du gouvernement fédéra1" le montre assez clairement (quarante-quatre au total). On qualifie les derniers événements (les émeutes de Québec): de "fausse manoeuvre" du gouvernement fédéral. La conscription fut "une lo1 adoptée malgré nous". Les désordres furent "provoqués par de sales agents bien payés". On parle même de "procédé lgnoble". Tel est le ton général du discours.

On ne donne aucun encouragement à nulle forme de violence. On la condamne même avec énergle. Les "briseurs de vitres, les assommeurs de soldats et les dévaliseurs de magasins" sont clairement dénoncés dans leur action. Au niveau de l'éloignement, on peut déjà émettre des réserves sérieuses. En effet, a cette époque on note une information écrite "assez objective" qui fait état de foule de 3000 manifestants et de divers saccages. Le Saguenay-Lac Saint-Jean n'était plus à ce moment un espace clos. 
Pour le langage, on peut affirmer que les deux journaux ont un discours assez virulent envers la politique gouvernementale fédérale. Le message est le même: Le gouvernement fédéral se trompe mais 11 faut éviter la lutte armée. On retrouve des mots comme: subissons, lutter dignement, patriotisme et traités brisés dans cette croisade contre la conscription et la loi martiale à Québec.

C'est un discours relativement cohérent, articulé et pondéré. Le fait intéressant, c'est de voir sa place dans la presse régionale. On le retrouve toujours en première page, rien de moins. On peut donc considérer que cet événement a revêtu un tntérêt considérable pour 1a presse régionale. Les émeutes de Québec ont été dêcrites comme un deuil national.

Nous avons rédigé cinq catégories pour analyser le message: vocabulaire désapprouvant 1a politique du gouvernement fédéral, manifestation d'autorité, représentants des forces de l'ordre, vocabulaire relevant d'une agression collective et encouragements a la violence. Dans 1a catégorie: vocabulaire désapprouvant la politique du gouvernement fédéral; le mot qui revient le plus souvent c'est fausse manoeuvre. On retrouve d'allleurs tout un vocabulaire qui va avec une telle affirmation: 
contre nous, abus d'autorité, profané l'exercice du droit de suffrage, traités brisés, etc. Ces termes pris dans un certain contexte rejettent l'entière responsabilité des événements sur le dos du gouvernement fédéral. Les éléments de cette catégorie ont une fréquence de 44 . La rubrique "mantfestation d'autorité" a une fréquence de 48. L'expression "1oi martiale" revient a 18 reprises. Cette catégorie cumule une deuxième place derrière la catégorie appelée "représentants des forces de 1'ordre".

On retrouve dans cette division des appellations comme: gouvernants, députés et politiciens, policiers, soldats, militaire et m11itaires, etc. Ces mots sont des synonymes des forces de 1'ordre qu'on devait s'attendre à rencontrer dans une pareille conjoncture. Les agents du pouvolr civil y sont tous compris (même le premier Ministre est cité).

Dans la catégorie "vocabulaire relevant d'une agression collective", on voit tous les mots qui témoignent d'un traumatisme de ce genre. Les termes apparentés a manifestations et émeutes reviennent le plus régulièrement avec une fréquence de douze chacun. Les adjectifs qui vont avec ce type d'événements y passent au complet: gravité exceptionnelle, 
regrettables, conséquences fâcheuses, spectateurs horrifiés, désordres, guerre, etc. Les expressions qu'on retrouve ordinairement dans 1a description d'un parell drame.

Finalement, la catégorie "encouragements à la violence" ne possède aucune fréquence. Telle est la conclusion qui se dégage de notre étude. Maintenant $s i$ on fait une récapitulation générale au niveau de $1 a$ signification du message analysé, on notera cect: les média écrits saguenéens désapprouvalent la politique du gouvernement fédéral mais ils n'étaient pas pour autant en faveur d'une action violente. De plus, 1ls ont ressenti les émeutes de Québec d'une certaine manière comme une agression collective envers eux.

Si on falt un résumé global au niveau des fréquences on arrive à ces résultats: La catégorie "représentants des forces de l'ordre" a la part la plus importante avec 61. Vient en deuxième place "manifestations d'autorité" avec 48. En troisième position sur un pled d'égalité arrivent ensuite les rubriques "vocabulaire désapprouvant la politique du gouvernement fédéral" et "vocabulaire relevant d'une agression collective" avec 44. Finalement "encouragements à la violence" obtient un zéro. Un dernier tableau illustre le classement. 


\section{TABLEAU VIII}

\section{Classemaent Par catÉgorie}

1. Représentants des forces de 1'ordre

2. Manifestations d'autorité

3. Vocabulaire désapprouvant la politique du gouvernement fédéral

3. Vocabulaire relevant d'une agression collective

4. Encouragements à la violence 
CHAPITRE II

LE PLÉBISCITE DE 1942

DANS LA PRESSE DU SAGUENAY 
Le premier septembre 1939 la Deuxième Guerre Mondiale commence. Le Canada est indirectement en conflit avec 1'Allemagne à cause de son appartenance à 1'Empire Britannique. Pour financer son effort de guerre le gouvernement fédéral décide de contrôler tous les emprunts. On crée une commission pour remp1ir cette fonction. C'est le début des hostilités au Canada. Les dépenses militaires ne devaient servir qu'à la défense du Canada. Le premier ministre King le dira. "Il Indiqua que la tâche du Canada serait naturellement d'assurer la défense de son propre territoire et de ceux du Labrador et de Terre-Neuve, en donnant une alde économique à 1'Angleterre." (1) Malgré tout cela, il y avait déjà une certaine contestation dans les rangs des libéraux à ce sujet. Personne ne voulatt la conscription pour le service outre-mer. Le quatre septembre le gouvernement King proclamait la Loi des mesures de guerre. Le consensus était brisé.

En effet, certaines personnes considéraient qu'ottawa ne pouvait pas faire adopter la Loi sur les mesures de guerre sans recourir à un plébiscite. A ce sujet, 11 y eut bientôt des pétitions et de nombreuses manifestations. Ce n'est que le neuf septembre que le ministre Ernest Lapointe expliqua les raisons de l'entrée en guerre du Canada.

1 - Mason Wade, Les Canadiens França1s de 1760 à nos jours, Ottawa, Le Cercle du Livre de France, 1963, Tome II, p. 345 
Une élection se jouera aussi sur le thème de 1'autonomie provinciale. Les polfticiens provinciaux et fédéraux sont allés très loin dans 1'affrontement verbal à la faveur de cette élection. Par exemple, on compare Duplessis à Hitler (à cause de la guerre éclaíre du second). Le fédéral décidera de se mêler a l'affaire, car c'est en fin de compte la politique internationale du gouvernement king qui est remise en jeu dans cette élection. Le gouvernement fédéral se substituera donc en grande partie au parti d'Adélard Godbout. "Adélard Godbout, embarqué dans le même bateau, ne pourra plus, pour son malheur, en sortir et devra servir de mousse au capitaine King-Lapointe." (2) C'est ce qui allait causer la perte de Maurice Duplessis. La possibilité d'une démission massive des libéraux en cas de victoire de Duplessis était perçue comme une catastrophe par la majorité des Québécols. Cela pour une raison: les libéraux provinclaux et fédéraux se présentalent comme le rempart contre la conscription. C'est le facteur déterminant de cette campagne.

Cependant 1 'homme qui fut le véritable artisan de cette victoire libérale a été Ernest Lapointe. C'est lui qui fit pencher la balance en faveur de son camp. Il aura été le grand stratège et le chef véri-

2 - René Chaloult, Mémolres politiques, Ottawa, Editions du jour, 1969, p. 112 
table des libéraux du Québec. Il se proposa comme le mur véritable contre la conscription. La défaite de Maurice Duplessis fut écrasante. Les libéraux s'engagèrent solennellement à démissionner si un seu1 Canadien français êtait mobilisé contre son gré en Europe. Après cette élection la position des deux partis est donc complètement Inversée. Montréal n'a élu que des libéraux. La presse anglophone a même été sans pitié. La presse anglaise et américaine considérait ce scrutin comme un gain pour les alliés. Le New York Times comparait Duplessis à Mussolini. A les lire, on pourrait presque penser que le sort du conflit mondial se joualt au Québec et non pas en Europe.

En 1941, tout le Canada est porté au découragement. Les alliés sont en difficulté. La conscription en vigueur depuis 1940 n'est pas totale et un mouvement d'opinion secouait encore les Canadiens. L'histoire ne se répète pas en général mais elle est prise quelquefols de sérieux bégalements. Encore une fols ce sont les conservateurs qui ouvrent le débat. "A 1a tête de ce mouvement d'opinion, on retrouve Arthur Meighen, 1'auteur de 1a lot de 1a conscription de 1917 et deux fols premier ministre conservateur pendant les années vingt." (3)

3- J.L. Granatstein, "Le Québec et le plébiscite de 1942 sur 1a conscription, Revue d'histoire de 1'Amérique Française, vol. 27, no 1 (juin 1973), p.73 
Lui et ses partisans voulaient, selon leurs mots: "Un effort de guerre total". Les libéraux de Mackenzle King se rappellent encore trop bien ce qui est arrivé lors de la première conscription pour le service outre-mer (division du pays, désordres, émeutes et isolement du Québec). King croyait que son parti étalt le seul quil pouvalt sauvegarder "1'unité canadienne pendant la guerre". King devait se battre sur deux fronts (à l'intérieur dans son propre cabinet et a l'extérieur contre les conservateurs et leur presse). Déjà le ministre de la défense nationale, J.L. Ralston, étalt favorable a la conscription outre-mer. Certains en reviennent même aux idées de 1917 . Sous les diverses pressions, King décidera de tenir un plébiscite. Les ministres québécois étaient d'ai1leurs de son côté. La conscription ne serait appliquée que si le recrutement volontaire s'avérait un échec. Il voulait avoir 1'avis aussi des électeurs par ce procédé.

Les anticonscriptionnistes, eux, avaient comme voix, 1a Ligue pour la Défense du Cananda ou LPDC. Pour certains d'entre eux, l'idée même d'un plébiscite était la rupture d'un contrat existant entre le Canada françals et le Canada anglais. La Ligue utilisa les mêmes moyens que les organisations de pression adverses. Georges Pelletier, directeur du Devoir faisait partie de la Ligue. D'allleurs ce journal la supporta constamment: "Only Le Devoir was enthusiastically campaigning for a 
"Non" vote". (4) I1 y avait une section à Chicoutimi, elle se réclamait de Henri Bourassa.

L'action de la Ligue avait ses limites car son budget était très restrelnt en comparalson avec celui d'Ottawa. De plus, au nfveau de la presse nationale, les journaux anglophones n'en parlèrent même pas. Le reste de la presse française (sauf Le Devoir) resta résolument neutre dans ce conflit. Malgré tout, la Ligue aura un succès certain. Les Québécols n'auront jamals été aussi unis sur une question. En avril 1942, selon Ga11up, $81 \%$ des gens du Québec s'élevalent contre la conscription. C'est un consensus très fort.

Le lundi solr, 27 avril 1942, les résultats seront les suivants: $63.7 \%$ pour le oul et $36.3 \%$ pour le non. Si on simplifie les positions, on arrive a cette conclusion: le Canada francais est pour le non et le Canada anglais est pour le oul. "The plebiscite results, however, demonstrated that the two nations of Canada each had their own-and

4 - J.L. Granatstein, Canada's War. The Polltics of the Mackenzie King Government 1939-1945, Toronto, Oxford Untversity Press, 1975, p. 223224 
separate-unity". (5) Les électeurs de Chicoutimi ont voté pour le non a $91 \%$. C'est d'allleurs 1'une des plus importantes proportions au Québec.

C'est un échec personnel pour King et un désavoeu de sa politique étrangère. Néanmoins, le Québec était moins isolé que lors de la première conscription. La blessure fut donc moins profonde. La conscription fut néanmoins retardée a cause de cela. Cette situation ne servira guère les intérêts des libéraux provinciaux.

L'assemblée provinciale est dissoute 1 e 28 juin 1944 . Des élections sont annoncées pour le huit août. La campagne sera difficile pour Duplessis. Il fut hué par des manifestants à plusieurs de ses as semblées. A Chicoutimi, le 21 juillet 11 quitta les lieux sous la protection de la police. Le Devolr n'appuyait pas Duplessis. On peut même dire que dans son ensemble la presse luf restalt résolument hostile. Duplessis se disait non séparatiste mais 11 voulait stmplement "être maitre chez nous", selon ses mots. Il allait bâtir sa campagne à partir

5 - J.L. Granatstein et J.M. Hitsman, Broken Promises: A History of Conscription in Canada, Toronto, Oxford University Press, 1977, p. 171 
de cette dernière affirmation. Duplessis rassurait car le Bloc populaire paraissait trop radical a la population canadienne française et le parti libéral de Godbout semblatt trop solfdaire du gouvernement d'ottawa. "Duplessis succeeded in making the federal government's policies towards the war and conscription the main issue of the election". (6) On a1lait le lui rappeler d'allleurs assez régulièrement, en particulier à Chícoutimi.

Analyse du contenu des journaux locaux

La majorité du non fut dans la région du Saguenay-Lac SaintJean écrasante lors du plébiscite du 27 avri1 1942 (91 \% dans le comté de Chicoutimi et $95.67 \%$ au comté du Lac Saint-Jean). Ce fut une victoire totale pour les partisans du non. Quelle a été la part du oul et du non dans L'Etoile du Lac et Le Progrès du Saguenay. Les deux journaux à cette époque étalent encore des hebdomadaires. Mais ils avaient ensemble un tirage de 7000 copies approximativement. Les deux journaux analysés touchaient de ce fait la majorité de la population saguenéenne. La question que nous allons nous poser est la suivante: La représentation du non

6 - Herbert F. Quinn, The Union Nationale Quebec: Québec Nationalism from Duplessis to Lévesque, Toronto, University of Toronto Press, 1979, p. 111 
a-t-elle été nettement plus forte que celle du oul dans les journaux régionaux?

Le journal local est un médium particulièrement important à ce moment. En effet, la télévision n'existait pas et la radio était un luxe dans plusieurs foyers saguenéens. Si le gouvernement fédéral a sous-estimé cette option c'est peut-être ce qui explique ses dêboires dans notre région. C'est un élément majeur selon nous qu'il faut prendre en compte dans une analyse d'une campagne politique.

Les deux journaux analysés sont: "Le Progrès du Saguenay" et "L'Étolle du Lac". Même si "L'Étoile du Lac" a gardé sa fonction d'encourager la colonisation par tous les moyens possibles, on peut dire que son contenu est nettement plus élaboré politiquement. Les articles qui parlent de politique cependant se retrouvent, à une exception près, dans "Le Progrès du Saguenay".

"L'Étolle du Lac" est de ce fait un journal largement plus "d'actualité". La couverture de l'événement (le plébiscite de 1942) par 
rapport a la première analyse de contenu s'est améliorée grandement. On peut affirmer que c'est une progression intéressante.

D́ans l'échantilion choisi on retrouve des éditoriaux, des lettres au journal, des retransmissions de discours et même des articles de propagande. Les deux journaux sont des émetteurs et les récepteurs furent les gens du Saguenay. La grande question est: Qui a eu le mellleur dans le débat?

Au niveau de la méthodologie, nous avons respecté le procédé utilisé antérieurement dans le premier chapitre. Notre échantillon est la totalité des documents portant sur le plébiscite de 1942 et les commentaires jugeant après-coup cette consultation populaire. C'est une analyse dirigée dans le sens que nous tentons de vérlfier ou d'infirmer une hypothèse solt: La représentation du non a étẻ beaucoup plus importante dans la presse régionale que celle du ouf. 
Notre étude porte sur 27 articles 17 dans Le Progrès du Saguenay et 10 dans L'Étolle du Lac. Notre travail porte sur une période de deux mols (avril et mal). Le fond est beaucoup plus gros que lors de la première analyse. En effet, certalns articles ont des pages entières et occupent le plus souvent la première page. C'est donc une source majeure sur laquelle 11 faut compter désormais. On retrouve dans les pages suivantes les tableaux représentant les catégories choisies alnsi que leur fréquence par journal. 
TABLEAU I

\section{CATÉgORIES}

VOCABULAIRE POUR LE NON

VOCABULAIRE POUR LE OUI

REPRESENTANTS DES FORCES DE L'ORDRE

VOCABULAIRE RELEVANT D'UNE AGRESSION COLLECTIVE

ENCOURAGEMENTS A LA VIOLENCE 
74 Vocabulaire pour le non

19 Vocabulaire pour le out

117 Représentants des forces de 1'ordre

0 Vocabulatre relevant d'une agression collective

2 Encouragements à la violence

100 Vocabulaire pour le non

32 Vocabulaire pour le oui

155 Représentants des forces de 1'ordre

0 Vocabulaire relevant d'une agression collective

2 Encouragements a la violence 
TABLEAU III

\section{VOCABULAIRE POUR LE NON}

MOTS

FRÉQUENCES

Votez contre la conscription

Répondons non

Faisons une croix vis-à-vis du mot "non"

Jamais

Votez pour la négative

Le pays sera divisé en cas de victoire du oul

Le contrat ne doit pas être brisé ou violé

Des engagements du gouvernement fédéral

Nous répondrons: "NON"

I1 faut répondre non

Nous sommes pour 1e non

Ne les écoutons pas

Votez - Ne pas craindre de voter NON

Votez non

Écrire le mot non sur votre bulletin de vote

Non

Partisans du non

La conscription pour outre-mer, nous n'en voulons pas

At tendu que...

Taxe de sang

Nouvel assaut contre la conscription 
TABLEAU IV

VOCABULAIRE POUR LE OUI

MOTS

FREQUENCES

oui

51

51 


\section{TABLEAU V}

\section{REPRESENTANTS DES FORCES DE L'ORDRE}

MOTS

FRÉQUENCES

Politiclens canadiens (J.E. Grégolre, Roland Angers, Maxime Raymond, Dr Philippe Hamel, Edgar Claveau, Louis-Joseph Lévesque, Laval Moffatt, Dr F.A. Dumas, M. Chaloult, Antonio Talbot, Godbout, Oscar Drouln, Ernest Lapolnte, M. King, J.E.A. Dubuc, Cardin, Borden, Meighen)

Politiciens étrangers (Roosevelt, Churchi11 et Hitler)

Chambre de commerce

Consells municipaux

Syndicats catholiques

Partl tory

Créditistes

Communistes (Tim Burke, son chef)

Directeur du Devoir (Georges Pelletier) 
TABLEAU VI

VOCABULAIRB RELEVANT D' UNE AGRESSION COLLECTIVE

MOTS

FREQUENCES

Aucun

0

TOTAL 


\section{TABLEAU VII}

ENCOURAGEMENTS A LA VIOLENCE

MOTS

Ut1liser la force

TOTAL
FREQUENCES

4

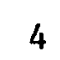


Résultats de l'analyse de contenu

A la lumière de cette enquête, on peut affirmer que la représentation du non dans les journaux réglonaux a été à l'image de sa victoire. Un trlomphe impresslonnant, et cela sans faire appel à la violence. En effet, la catégorie: "encouragements à la violence" ne recueille que 4 mentions. De plus, c'est le camp du non qui accuse le camp du oui de vouloir utiliser cette option en cas de défalte. En aucune manière on ne veut s'attaquer par la force au "déroulement démocratique" du scrutin.

La catégorie "vocabulalre pour le non" obtient un total cons1dérable de 174 fréquences. C'est un fait quil montre bien la conjoncture polltique existant dans 1a presse écrite du Saguenay- Lac SaInt-Jean de cette époque. On s'insurge en bloc contre "une possibilité de conscription outre-mer". Les épithètes pleuvent littéralement. La Ligue pour la défense du Canada considère qu'il existe un contrat entre le gouvernement fédéral et ses c1toyens et que le seul falt de le remettre en question par un plébiscite est tout simplement une action inacceptable. Les conseils municipaux de la région sont tous contre cette consultation. Un éditorialiste (dans un article qu'on retrouve dans Le Progrès du Saguenay

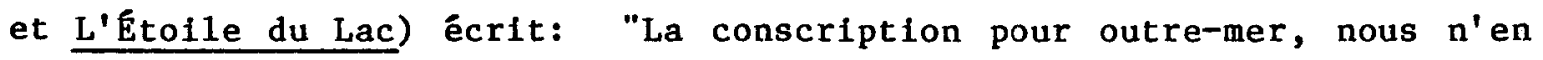
voulons pas". De plus, les orateurs pour le non ont des pages entières de publicité. 
La position du Conseil Central des Syndicats Nationaux Catholiques du diocèse de Chicoutimi renforce ce réquisitolre en faveur du non. Une résolution est adoptée et elle demande à tous les ouvriers du diocèse de voter non au plébiscite. On exhorte aussi les gens à "ne pas cralndre de voter non". Un journaliste considère que "c'est la seule réponse qui soit digne a la question".

Quant a la Chambre de commerce de Charlevolx-Ouest, elle demande justice pour les partisans du non. Un peu plus tard, c'est le comité régional de la Ligue pour la défense du Canada qui demande au député fédéral J.E.A. Dubuc pourquol il a voté en faveur de la loi du plébiscite et 11 l'encourage à "faire connaitre ouvertement et publiquement son attitude sur 1a question du 27 avri1".

Le député provincial de Chicoutimi, Antonio Talbot, est le plus dur envers le gouvernement fédéral. Il répondra "un non catégorique" selon ses propres mots. De plus, 11 cite abondamment, pendant deux pages entières, Ernest Lapointe et le premier ministre (Mackenzie King) pour appuyer sa position. Après une pareille démonstration, i1 ne faut donc pas s'étonner de la victoire écrasante du non dans la région. 
Quant à la représentation du oul, on dolt remarquer que cette catégorie ne recuel11e que 51 fréquences. Les défenseurs de cette option n'ont jamais fait sentir leur présence dans les journaux régionaux. C'est même le camp du non qui en fait mention dans sa campagne. On peut affirmer que les défenseurs du oui n'ont guère fait sentir leur poids dans les journaux locaux. Leur présence a même été inexistante. Leur défaite dans les média écrits du Saguenay était certaine.

La catégorle: "représentants des forces de l'ordre" récolte la plus grande fréquence soit 272. Voici la raison: le camp du non cite à tout propos les déclarations des politiclens fédéraux au sujet de $1 a$ nonimposition de la conscription (Lapointe et King plus particulièrement). On se sert de l'enneml (le fédéral) pour renforcer sa position.

Si on falt état d'autres points de vue, on parle des orateurs qui sont pour le non: les Maxime Raymond, Dr Philippe Hame1, Antonio Talbot et compagnie. On volt que cette catégorie a servi, en majorité, les partisans du non. Le camp du non avait un atout supplémentaire. La catégtorie "vocabulaire relevant d'une agression collective" n'obtient aucune fréquence. Ce facteur tendrait à prouver qu'on considère ce scrutin "comme une élection ordinaire". On veut un non fort pour avertir 
le gouvernement fédéral, selon les mots de la Ligue pour la défense du Canada, que le Québec ne tolérait pas une autre conscription outre-mer.

Finalement la partie du travail "encouragements à 1a violence" ne possède que quatre fréquences. Le terme employé est le mot force. C'est, rappelons-1e, le camp du non qui accuse son rival de vouloir 1'utiliser. On semble résigná à combattre par les voies légales et démocratiques. Si on fait une récapitulation générale au niveau de la signification du message analysé; on notera ceci: le camp du non a occupé complètement la presse régionale (peut-être parce que c'était le seul espace qui lui restait). Le camp du non a joué cette carte magnifiquement. Dans son discours, 11 n'a jamais été question d'utiliser la violence. Cependant, les média écrits saguenéens n'ont pas ressenti cet événement (le plébiscite) comme une agression collective.

Au niveau des fréquences, on arrive à ces résultats: 1a catégorie "représentants des forces de 1'ordre" obtient le nombre de fréquences le plus élevé solt 272. La deuxième place appartient à "vocabulaire pour le non" avec 174. La troisième place est tenue par la rubrique "vocabulaire pour le oul" avec 51. La quatrième position est la propriété de la catégorie "encouragements à la violence" avec quatre. Finalement "vocabulaire relevant d'une agression collective" a un zéro. Un dernier tableau qui illustre la situation. 
TABLEAU VIII

Classement par catÉgorie

1. Représentants des forces de l'ordre

2. Vocabulaire pour le non

3. Vocabulaire pour le out

4. Encouragements a la violence

5. Vocabulaire relevant d'une agression collective 


\section{CHAPITRE III}

LA MORT DE PIERRE LAPORTE (1970)

DANS LES MÉDIA ECRITS SAGUENEENS 
Le F.L.Q. se manifeste officiellement en 1963. Dans un communiqué, 11 se définit comme un mouvement révolutionnaire qui frappera le colonialisme anglais sous toutes ses formes et son allié naturel américain. I1 faut retourner à la Révolution tranquille, pour expliquer ce mouvement. Après seize ans de régime conservateur et nationaliste, Jean Lesage prend le pouvoir au Québec. Des changements surviennent bientôt. "Molns de trois mols après le changement de régime, le Rassemblement pour l'indépendance nationale (R.I.N.) voit le le jour". (1) Le R.I.N. veut aussi s'attaquer au colonfalisme, cependant, il cholsit l'arène politique pour falre triompher ses Idées.

Le monde est à 1'heure de 1'Algérie, de Cuba et du Viêt-nam. En Irlande, 1'I.R.A falt parler d'elle et, en Espagne, 1'E.T.A marque des points. Le F.L.Q. volera d'abord des explosifs. Une semaine avant les élections fédérales du premier avril, trois bombes sautent. Déjà certains journaux font l'analogle avec l'0.A.S. en Algérie (La Presse en particulier). Le 16 avri1 1963, 1e F.L.Q. 1ance son premier manifeste, c'est un appel à 1'insurrection. Dans la nuit du 20 au 21 avril 1963, 1e F.L.Q. falt son premier mort (le vellleur de nuit Wilfrid o'Ne11). Dans

1 - Louis Fournier, F.L.Q.: Histoire d'un mouvement clandestin, Montréal, Québec/Amérique, 1982, p. 17 
1a nuit du 16 au 17 mai à Westmount, 10 bombes explosent. A la suite de cet incident plusieurs militants du R.I. N. sont arrêtés. Pierre Bourgault et René Levesque condamnent cette action. Cependant à la fin de 1967, tous les premlers militants du F.L.Q. seront éliminés. Ils sont en liberté conditionnelle ou ils ont choisi 1'exil.

La violence dite "politique" était un phénomène nouveau à l'époque pour la police et la population canadienne. "Depuis aô̂t 1917, date a laquelle la demeure du propriétaire du Montreal Star, fut dynamitée par des gens qui voulalent protester contre la conscription, 11 n'y avait plus eu d'actes terroristes au Québec". (2) Ce sont des Québécois d'adoption quí ont été les premlers chefs de ces cellules. Le groupe de Françols Schirm, un immigrant d'origine hongroise, étałt composé de six personnes dont une étalt issue du Saguenay-Lac Saint-Jean.

De nouveaux réseaux viendront au monde: l'Armée de libération du Québec (A.L.Q.) et la Cognée. Idéologiquement on s'inspire des révolutions algériennes et cubaines. On se réclame aussi des patriotes de

2 - Gustave Morf, Le terrorisme québécols, Montréal, Éditions de 1'homme, 1970 , p. 9 
1837. A l'été 1963 , la violence refait surface. Et l'A.L.Q. commence ses opérations de financement et de réquisitions de matérlel (vols de banques et d'armes). D'autres attentats ont lieu bientôt. Mais la police marqua un important point le 9 avril 1964 en neutralisant le noyau de 1'A.L.Q.. Le mols de mal allait être encore plus chand. Lors de 1a fête de 1a Reine Victoria (Victoria Day) à Montréal, 85 personnes sont arrêtées (en majorité des indépendantistes du R.I.N.). Juin 1964, un nouveau groupe prend la relève: l'Armée révolutionnaire du Québec. Un raid à 1'International Firearms fera deux morts, mais les participants de cette action auront des peines très sévères. Une connexion Alger-Québec apparait. Le 10 octobre 1964, 1a Reine Elisabeth II viendra au Québec. Des mesures de police exceptionnelles seront prises et se solderont par le tristement célèbre "samedi de la matraque".

Le 23 septembre, 1e premier ministre Pearson décide de mesures d'espionnage vis-à-vis du mouvement indépendantiste et du R.I.N. en particulier. On compare ce dernier parti au communisme. Le 15 décembre, La Cognée rend publique la création de comités régionaux. Un comité est situé au Saguenay-Lac Saint-Jean. Notre réglon semblait être un endroft stratégique que le F.L.Q. voulalt investir. On a vu qu'11 y avalt des membres des premières cellules qui provenalent de la région, cela sans compter les membres "potentiels" et ceux qui sont restés dans 1'anonymat. En 1965, une filiation apparaft entre le F.L.Q. et le "Black Liberation 
Front". Un complot de dynamitage existait entre eux. L'affaire aura un grand retentissement. De plus, on commencera à toucher aux intérêts américains. C'est le début d'une série d'attentats. Ce sera un été chaud. Notre région sera encore touchée: un vol de 1000 bâtons d'explosifs à 1a compagnie de papier Price d'Alma.

Le plus spectaculaire restait à venir. Un commando avait la tâche de s'attaquer à une base militaire importante. La Macaza, une base mllitaire installée dans la forêt des Laurentides près de Mont-Laurier, était 1'un des points stratégiques du système de défense de 1 'Amérique du Nord (N.0.R.A.D.). La tentative avorta, mais le rôle de notre région fut mis encore en valeur: une cellule étalt du Saguenay. Elle était formée de trols jeunes d'Alma. Un peu plus tard la police réalisera un coup d'éclat en démantelant une cellule du F.L.Q. reliée au réseau de La Cognée. Ce groupe avalt des liens "intimes" avec notre région.

A 1'automne 1968, on assiste à la création du Parti québécois. Pendant 1'été 1969, attentats (certains ne seront jamais éclaircis) et protestations populaires troublent 1'atmosphère. Notre région sera touchée par le phénomène: 250 bâtons de dynamite sont trouvés la veille de 
1'arrivée du premier ministre Trudeau à Roberval. Le contexte international de 1'époque étalt particulièrement chargé. Ce n'était donc pas un cas unique au monde. En 1969, 11 se prodult une vague d'enlèvements politiques en Amérique du Sud (1'ambassadeur des Etats-Unis à Rio de Janeiro entre autres). On pouvalt s'attendre logiquement à des actions de ce type si une crise politique et sociale se manifestait dans un avenir rapproché au Canada. "Les conflits intérieurs violents subissent l'Influence de la société Internationale".

De plus, il se produit une escalade verbale dangereuse. Le ministre provincial de la justice déclare qu'il existe près de 3000 terroristes au Québec. La G.R.C., dans un rapport, soutient que l'agitation politique est faite a l'étranger (Cuba, l'Algérie et même Moscou). Encore une fois à cette époque, certaines cellules de Montréal ont des contacts étroits avec notre région. Le F.L.Q. étalt donc très présent dans notre région (1'histoire de ce mouvement le prouve). Si son action a été plus spectaculalre a Montréal, c'est pour des raisons stratéglques et tactiques.

3 - Marc Laurendeau, Les Québécols violents: un ouvrage sur les causes et la rentabilité de la violence d'inspiration politique au Québec, Montréa1, Éditions du Boréa1 Express, 1974, p. 199 
A 1'aube de la crise d'Octobre 1970, le groupe de Paul Rose était le mieux organisé et préparé des cellules du F.L.Q.. La ronde des attentats recommence. Un complot pour enlever un représentant officiel des Etats-Unis au Québec est éventé de peu. Notre région est encore touchée par le phénomène: un membre du F.L.Q. est arrêté et condamné à trois ans de prison. A ce moment les effectifs du F.L.Q. sont approximativement de 35 personnes. Au début du mols d'octobre, tout se précipite lorsqu'on enlève le diplomate britannique James Richard Cross, un lundi 5 octobre. L'opération est parfaitement réussie. Le manifeste du F.L.Q. est lu sur les ondes de Radio-Canada. Ottawa refuse de négocier. Le samedi 10 octobre, Pierre Laporte est kidnappé devant sa demeure. La G.R.C. rédige une liste de gens à arrêter prioritairement. Elle est prête pour les mesures de guerre. Dans un premier temps, 8000 soldats sont envoyés à Montréa1. Un peu plus tard, à la demande des autorités provinciales, les mesures de guerre sont amorcées le vendredi 16 octobre 1970 à 4 heures du matin. Les chiffres parlent d'eux-mêmes: 500 citoyens arrêtés, 4600 perquisitions avec saisies et 34700 perquisitions au total. Le Saguenay et 1'ensemble du Québec furent touchés.

On refuse officlellement la négociation. C'est ce qui entraînera la mort de Pierre Laporte, le samedi 17 octobre, en fin d'après- midi. Elle sera connue à minuit. René Levesque condamne violemment cette action. Lui et Claude Ryan lancent un appel au gouvernement pour qu'- 
il négocie pour sauver la vie de James Cross. On négociera avec les ravisseurs et James Cross sera épargné. Le F.L.Q. ne retrouvera plus sa puissance d'autrefois. La plupart de ses militants sont en exil. De plus, la police a eu le temps de l'infiltrer en grande partie. En 1971, son activité est en nette régression. L'état d'exception prend fin le 30 avril.

"Les opérations de déstabilisation" se succéderont. La G.R.C. volera la 1iste des membres du Parti québécois (100 000 membres). Le 15 novembre 1976, le P.Q. est porté au pouvolr. Les exilés (ex-membres du F.L.Q.) commenceront à rentrer au pays. Cette crise lafsse beaucoup de questions en suspens. Elle a favorisé un mouvement vers la droite. "Je n'ignore pas que les événements d'octobre ont engendré un climat de tension et de peur, qu'lis ont favorisé un glissement vers la droite, vers 1e Law and Order". (4)

Le risque d'un mouvement clandestion du type terroriste semble toujours présent. Le danger que pose ce genre d'action sur une démocra-

4 - Gérard Pelletier, La crise d'octobre, Montréal, Éditions du jour, 1971 , p. 173 
tie comme la nôtre est important. "Le consensus démocratique est fragile; il l'est par essence". (5) Si une nouvelle crise d'Octobre arrivait demain, on peut se demander sérieusement si les mêmes erreurs d'interprétation de part et d'autre (1'appareil judiciaire et gouvernemental) ne se reproduiraient pas. La police suivrait-elle toujours la légalité? On peut en douter également en lisant la conclusion de la commission Keable. "En dernière analyse, seuls 1'Etat et son appareil policier profitent du terrorisme". (6)

Analyse de contenu des journaux locaux

L'assassinat de Plerre Laporte fut le point culminant de la crise d'Octobre 1970 et une étape significative dans 1'histoire du terrorisme au Québec. Il choqua profondément 1'opinion publique. La condamnation de cet acte fut générale et très violente "verbalement". Le F.L.Q. à partir de ce moment a commencé son déclin. Cette condamnation morale a-t-elle été très importante dans les journaux régionaux? Nous

5 - Fernand Dumont, La vigile du Québec Octobre 1979: 1'impasse?, Montréal, Éditions HURTUBISE HMH, Ltée, 1971, p. 188

6 - Québec (Prov.), Commission d'enquête sur des opérations policières en territoire québécois, Québec, Ministère de la Justice, 1981, p. 440 
pensons que oui. C'est ce que nous allons examiner précisément. Quelle a été la part de réprobation dans Le Soleil, L'Étoile du Lac et Le Progrès-Dimanche? A-t-elle été unanime? Quí 1'a faite?

Le Solefl étalt un quotidien et les deux autres journaux des hebdomadaires. Le quotidien analysé avait une partie appelée: Le Solel1 du Saguenay-Lac Saint-Jean depuis 1946. Une large part de sa production était donc exclusivement consacrée à notre région. Son influence étalt certainement très lourde étant donné sa position de monopole dans le domaine de l'information journalière. Le Progrès-Dimanche avait un tirage dépassant les 35000 exemplaires. Finalement L'Étoile du Lac obtenait un tirage de moins de 3000 copies.

Le Saguenay n'est plus coupé des grands centres. La téléviston est dans tous les foyers saguenéens et la radio n'est plus un luxe. Le $\underline{\text { Soleil }}$ et Le Progrès-Dimanche sont des journaux d'information. L'Étolle du Lac ne vante plus les mérites de la colonisation mais son contenu parle largement de l'agriculture. Son contenu"politique" est totalement différent des deux autres journaux ce qui est un falt nouveau. Dans 1'échantillon analysé on retrouve des éditoriaux, des lettres au journal, 
des retransmissions de discours, des condensés de presse étrangère, des Interviews et même une messe. C'est l'échantilion le plus important 70 articles et le plus intéressant en diversité que nous avons analysé jusque-1à. Notre échantillon est la totalité des documents portant sur le meurtre de Pierre Laporte en octobre 1970.

Au niveau de 1a méthodologie, nous avons respecté le procédé utilisé antérieurement dans les chapitres précédents. C'est une analyse dirigée dans le sens que nous tentons de vérifier ou d'infirmer une hypothèse solt: Que la réprobation du meurtre de Plerre Laporte a été totale et très violente dans la presse écrite régionale. Cet événement quí s'est produit à Montréal (la mort de Pierre Laporte) aura une incidence majeure au niveau local (notre région). Les grands moyens de communication (presse, radio et télévision) sont beaucoup plus puissants. Notre étude portait sur 70 articles (65 dans Le Soleil, deux dans L'Étolle du Lac et trois dans Le Progrès-Dimanche). Notre travail portait sur une période d'un mois. On retrouve dans les prochaines pages des tableaux représentant les catégories retenues ainsi que leur fréquence par journa1. 
TABLEAU I

CATEGORIES

VOCABULAIRE DÉSAPPROBATEUR

VOCABULAIRE DESAPPROBATEUR TRÈS VIOLENT VERBALEMENT

IDENTITE DES ACTEURS

REPRÉSENTANTS DES FORCES DE L'ORDRE

VOCABULAIRE RELEVANT D'UNE AGRESSION COLLECTIVE 
98 Vocabulałre désapprobateur

72 Vocabulaire désapprobateur très violent

220 Identité des acteurs

53 Représentants des forces de 1'ordre

9 Vocabulaire relevant d'une agression collective

1 Vocabulaire désapprobateur

1 Vocabula1re désapprobateur très violent

1 Identité des acteurs

0 Représentant des forces de 1'ordre

0 Vocabulaire relevant d'une agression collective

1 Vocabulaire désapprobateur

2 Vocabulaire désapprobateur très violent

2 Identité des acteurs

1 Représentants des forces de l'ordre

o Vocabulaire relevant d'une agression collective 


\section{TABLEAU III}

\section{VOCABULAIRE DESAPPROBATEUR}

MOTS

FREQUENCES

Assassin (s), assassinat ( 8 ), assassinat (s) politique ( $s$ ), assassiné

Atterré, apprêhende une révolte 2

Conspirateurs, criminels, condamne, condamnation, condamné 6

Exécution, émotion, éclaboussé $\quad 6$

Fanatiques, forfait, forfaitures 3

Gangstérisme, geste déplorable 2

Héros, horreur $\quad 6$

Indignation, Innocent 4

Martyr (s), mort tragique, meurtre (s), meurtriers 21

Perte inestimable, profondément choqué 2

Relève du droit commun, réprobation unanime, réprouvé
cette action

Tristesse, terroristes 3

$\begin{array}{ll}\text { TOTAL } & 100\end{array}$ 


\section{TABLEAD IV}

\section{VOCABULAIRE DESAPPROBATEUR TRES VIOLENT}

MOTS

FREQUENCES

Assassinat ignoble, organisé, acte barbare, de

barbarie, acte absurde et méprisable

L'assassinat d'innocents, action injustifiable, action qui déshonore 1'espèce humaine, agents du chaos, arrivés au crime, acte épouvantable et barbare

Actes de pirates, de ganster et de criminels de droit commun 1

Barbarie, bande d'assassin anonyme

Crime aussi affreux et 1âche, corriger des injustices par des injustices

Crapules, crime aussi inutile qu'impardonnable, crime ignoble, Indigne d'hommes civilisés, criminels barbares, $c^{\prime}$ est la loi de la guerre

Des actes aussi ignobles, des actes d'une cruauté aussi raffiné, dégoût

Êtres Inhumalns, effroyable et brutal assassinat, étouffé par l'émotion

Frustrés et des ratés, folle devenue furleuse

Geste criminel, geste aussi atroce et aussi sauvage, geste non seulement criminel mais insensé, gaspillage atroce

I1s l'ont tué en même temps que Plerre Laporte

Ils nous ont tous plus ou moins éclaboussés

La plus grande injustice, 1'ignominie d'un crime, lâcheté, 1'irresponsabilité des ravisseurs, les victimes sont innocentes

Le Québec ne sera plus jamais le même

Le pays est en guerre, 1'horrible choc de sa perpétration 
TABLEAU IV

VOCABULAIRE DESAPPROBATEUR TRÈS VIOLENT SUITE

MOTS

FRÉQUENCES

Meurtre inhumain, marqué au fer rouge de la honte et de l'infamie

M'écoeure, meurtre affreux et lâche, meurtres politiques

Meurtre brutal perpétré de sang frold, meurtre ignoble

Martyr de la démocratie

Ont quelque chose qui ne va pas dans le plafond

Par des gens sans âme, pas de mot assez fort pour condamner ce crime

Qu'on viole impunément ses 11bertés, guerre civile

Responsabilité collective

Sadisme, sont pas capables de falre leur vie, semant violence, la haine fanatisme, sursaut d'indignation et d'horreur, soumis cruellement à la torture, sacrifié injustement, tant de cruauté

Tueurs lunatiques, vlolence lunatique, un événement révoltant 


\section{TABLEAU V}

\section{IDENTITE DES ACTEURS}

MOTS

FRÉQUENCES

Agence de Presse (Londres, R.F.A., Autriche, Rome

Genève, Lima, Mexico, etc.)

Editorialistes (Gilles Boyer, Paul Lachance,

A. Tremblay, Raymond Dubé, Jean-Claude St-Pierre) 5

Elisabeth II

1

Gouvernements étrangers (Londres, Washington, Paris, Belfast, Vienne, Pretoria)

Gouvernements étrangers qui refusent de commenter (Alger, Moscou, La Havane, Belgrade, Pékin)

L'homme de la rue (6 étudiants, 2 inconnus, 2 traval1leurs,

1 imprimeur, une institutrice)

Maires du Saguenay-Lac Saint-Jean

Organismes internationaux: L'O.N.U. et C.I.C.R.

(Comité international de la Crolx-rouge)

Politiciens: Réal Caouette, René Lévesque,

Jean-Jacques Bertrand, Camil Samson, Robert Bourassa,

De Gaulle, etc.

Religleux: Mgr Grégolre, Cardinal Roy,

Mgr Russel F. Brown, Mgr Marius Paré, Paul VI 10

$\begin{array}{ll}\text { Journaux canadiens-angla1s } & 17\end{array}$

$\begin{array}{lr}\text { Journaux français } & 7\end{array}$

TOTAL 
Chef de 1'Union nationale 2

Gouvernement fédéral et québécois 20

Le ministre du traval1 et de l'immigration 12

Policiers ou sûreté du Québec 4

$\begin{array}{lr}\text { Premier ministre Trudeau } & 6\end{array}$

Premier ministre Bourassa 2

Parti québécois, chef péquiste 1

TOTAL 


\section{TABLEAU VII}

\section{VOCABULAIRE RELEVANT D'UNE AGRESSION COLLECTIVE}

Consternation générale

Désastre

Terrible tragédie

L'angolsse 
Résultats de 1'analyse de contenu

A la lumière de cette enquête, on peut affírmer que 1a réprobation du meurtre de Pierre Laporte a été unanime et imposante dans les journaux réglonaux. Le consensus se veut total dans la réprobation. Personne n'excuse le F.L.Q.. Personne ne se porte à sa défense. La dénonclation la plus énergique de ce mouvement vient de la part du chef péquiste René Levesque qui avalt problablement le plus à perdre. En aucune manière, on ne veut sanctionner ce type d'action.

D'ailleurs, il n'est pas surprenant que la presse régionale ait condamné le meurtre de Pierre Laporte. La presse du Canada entier l'a fait aussi. C'est le contraire qui l'aurait été. La situation analysée montre encore une fols que le Saguenay n'a pas souffert de pénurie d'information écrite. C'étalt le but de l'exercice. Les média écrits ont respecté la logique anticipée. Le message était le même qu'ailleurs.

La catégorie "vocabulaire désapprobateur" obtient un total de 100 fréquences. Si on lui additionne la catégorie "vocabulaire désapprobateur très violent" on arrivera au total de 175 .fréquences. Les mots ne seront jamais assez durs pour dénoncer les meurtriers de Plerre Laporte. Pierre Laporte fut "un héros et un martyr" selon certains. 
Pour d'autres, c'est "un assassinat ignoble, un acte barbare et un meurtre Inhumain". Pour Robert Bourassa selon ses propres mots, Pierre Laporte fut un martyr de la démocratle. Pour René Lévesque, les meurtriers de Plerre Laporte sont "des êtres inhumalns". "Ils ont tué leur cause en même temps que Plerre Laporte", dit-11. Ils nous ont tous plus ou moins éclaboussés" termine-t-11 finalement.

L'éditorialiste du Solell, Gilles Boyer, titre: "La folie devenue furieuse". On pourralt continuer ainst pendant des heures. La catégorle "identité des acteurs" obtient une fréquence de 223 . C'est la plus haute fréquence. L'identité des personnes qui réprouvent et condamnent le meurtre de Plerre Laporte est imposante et parle par elle-même. Le monde entier donne son commentaire. Au niveau des agences de presse, 1a majorité des pays occidentaux se retrouve dans l'exposé.

Certains des pays du tiers monde qui sont du côté occidental ont une place dans la condamnation générale (le Pérou et le Mexique). Cependant, 11 faut noter qu'un nombre assez important de gouvernements étrangers ont refusé de commenter 1'événement: 1'Algérie, 1'U.R.S.S., Cuba, 1a Yougoslavie et 1a Chine. Au niveau des gouvernements étrangers qui réprouvent officlellement cet acte de terrorisme, on retrouve comme 
par hasard les pays qui ont le plus à perdre par ce type d'action: 1'Angleterre, les États-Unis, 1a France, 1'Irlande du Nord et même 1'Afrique du Sud. L'homme de la rue est auss 1 représenté dans l'interprétation de cet événement dramatique: douze personnes sont interviewées.

Le réquisitoire réprobateur est écrasant. On retrouve aussi une présence marquée du niveau local. En effet, tous les maires du Saguenay-Lac Saint-Jean Iront de leurs remarques sur 1'affaire Laporte. Certaines seront très dures. Évidemment, les organismes internationaux auront leur mot à dire (1'O.N.U. et le Comité international de la CroixRouge). Les politiclens importants seront également cités: Réal Caouette, René Levesque, Jean-Jacques Bertrand, Camil Samson et Robert Bourassa. On cite même les propos de la Reine d'Angleterre.

Le monde religieux a aussi une place dans le discours condamnatoire: Mgr Grégoire, le Cardinal Maurice Roy, 1'Evêque ang1ican de Québec Mgr Russel F. Brown, Mgr Marius Paré et même le pape Paul VI. L'autorité religieuse est très lourde dans ce genre de moment. De plus, les journaux canadiens anglais sont mis a contribution: Le Telegraph-journal de Saint-Jean, N.-B., Le Globe and Mail de Toronto, Le Telegram de Toronto, etc. Les grands pérlodiques du pays y passent tous. Les fournaux 
françats aussi: Le Paris-jour, Le Parisien Libéré, Les Échos, Combat et

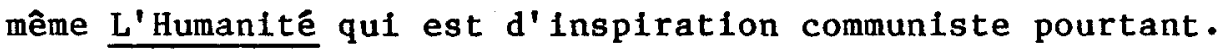

La catégorie "représentants des forces de 1'ordre" obtient un total de 54 fréquences. C'est relativement peu pour ce genre de crise. On semble faire plus appel à la réprobation individuelle qu'a 1'autorité pour condamner le meurtre de Pierre Laporte. Les titres: chef de 1'Union nationale, chef péquiste, premier ministre Trudeau et premier ministre Bourassa sont peu utilisés pour appuyer la réprobation. L'émotion populaire se traduit par une prise de position individuelle plutôt que par une prise de position institutionnelle.

La catégorie "vocabulaire relevant d'une agression collective" n'obtient qu'un pauvre neuf fréquences. Cette situation tendrait à prouver qu'on n'a pas considéré cette crise comme une agression collective. On 1'a ressentie plutôt comme une espèce de deuil national. Les mots cauchemar, consternation générale, désastre, terrible tragédie et angoisse peuvent très bien témolgner de cette situation. 
Maintenant, si on falt une récapitulation générale au niveau de la signification du message analysé, on arrivera aux conclusions suivantes: tout d'abord, 1a réprobation du meurtre de Plerre Laporte a été violente "verbalement". L'identité des acteurs de cette dénonciation fut très pesante dans le processus (de 1'0.N.U. jusqu'au maire de Jonquière). On n'a pas falt appel aux représentants des forces de 1'ordre mais à la consclence individuelle des participants. Les média saguenéens n'ont pas ressenti ce drame (1'affaire Laporte) comme une agression collective mais plutôt comme un deuil national.

Si on fait un résumé au niveau des fréquences, on arrive à ces résultats: la catégorle "vocabulaire désapprobateur" obtient le nombre de fréquences, le deuxième plus élevé, solt 100. La catégorie "vocabulałre désapprobateur très violent" arrive en trolsième place avec 75 . La première place est tenue par la catégorie "identité des acteurs" avec 223. La quatrième position est tenue par la catégorie "représentants des forces de 1'ordre" avec 54. Finalement "vocabulaire relevant d'une agression collectlve" arrive a la fin du peloton avec un neuf. Un dernier tableau qui illustre la situation. 


\section{TABLEAU VIII}

\section{CLASSEMENT PAR CATÉGORIE}

1. Identité des acteurs

223

2. Vocabulaire désapprobateur

3. Vocabulaire désapprobateur très violent

4. Représentants des forces de l'ordre

5. Vocabulaire relevant d'une agression collective 
CHAPITRE IV

LE REFERENDUM DE 1980 DANS LES

MÉDIA ECRITS DU SAGUENAY 
Le 15 novembre 1976, le Parti québécois prenait le pouvoir. On pouvait s'attendre à une consultation sur une question fondamentale. "Elle entrouvalt la porte de l'indépendance, dans la promesse même des péquistes de ne pas la faire d'emblée si le parti était au pouvoir". (1) En effet, les deux positions des diverses parties a l'origine semblalent être Irréconciliables. Pour M. Lévesque, 1'indépendance était devenue "irréversible". Pour M. Trudeau, la séparation était "impensable".

A cette occasion, le Québec revendiquait un nouveau statut. "Les québécois de langue française constituent maintenant une nation, indépendamment de la reconnaissance de ce fait par le reste du Canada". (2) Le mouvement indépendantiste étalt très récent. I1 existait depuis tout au plus une vingtaine d'années.

1 - Gérard Bergeron, Ce jour-1à... le référendum, Montréal, Quinze, 1978, p. 55

2 - Robert Boily et collaborateurs, Québec: un pays incertain, réflexion sur le Québec post-référendaire, Montréal, Québec/Amérique, 1980, 
Les premiers indépendantistes au sein du R.I.N. ne voulaient pas "d'une association avec le Canada anglais". On veut être désormais "maître chez-nous". En 1967, René Lévesque alors 1'une des grandes figures du parti libéral provincial propose un plan qui veut changer radicalement la position du Québec dans le Canada. Après un refus, 11 fonde un parti avec quelques fidelles: le mouvement souveraineté-association (M.S.A.) ou Parti québécois. Le Parti québécols occupera la position dominante dans le mouvement Indépendantiste. Son avance sera spectaculaire sur la scène politique. On ne parle pas seulement d'indépendance dans ce parti mais de souveralneté. On volt donc qu'à l'origlne le Parti québécois n'étalt pas le R.I.N..

Cette tactique a porté fruit politiquement durant la période 1970 a 1976. On peut définir le Parti québécois comme un parti centriste dans le mouvement indépendantiste. Après sa prise du pouvoir on pouvait logiquement s'attendre à ce que le Partí québécois se lance dans le projet qui l'avalt mis au monde: la souveralneté-association. C'est ce qui devait exactement arriver d'allleurs.

Le gouvernement a adopté la 10192 pour délimiter le jeu électoral. Les deux camps semblaient avoir au départ les mêmes chances. Le 
Parti québécols étant au pouvoir avalt quelques chances supplémentaíres en partant. On allait créer deux comités pour représenter les deux options: un pour le oul et un pour le non. Le comité du oui allait s'emparer du local en mettant 1 'accent sur 1 a formation de regroupements 1ocaux. Le Parti québécois parrainalt le comité du oui. Ce dernier n'était pas une formation politique officielle cependant. Le comité du non était une formation plus compliquée à cause des différents partis politiques qui prônaient le non.

I1 y aurait des différences dans 1'utilisation des média. Ce sont deux philosophies différentes représentant deux projets de société qui s'affrontent. Le gouvernement fédéral attendait de pied ferme le Parti québécois. Les autres provinces refusèrent toutes le projet de souveraineté-association du gouvernement Lévesque avant même de volr les résultats de cette consultation publique.

Dans ce débat, les partisans du non ont réussi à faire mieux valoir leur vision des faits: 1a souveraineté-association équivalait a l'indépendance complète du Québec. Les notions du public vont devenir confuses. Par exemple a un certain moment, un non deviendra oui. Un porte-parole du gouvernement fédéral promettait des changements constitu- 
tionnels si on répondait non à la question. "Pourtant, nous sommes convaincus qu'il faut profiter du climat actuel pour faire un ménage constitutionne1, au moins pour éliminer ce qui est désuet, comme le drolt de désaveu, ou consacrer ce quí est devenu une pratique courante, comme l'accès par les provinces a la taxation indirecte". (3)

On créa huit mille regroupements pour le oui. Ces regroupements de rues, de quartiers, de traval1leurs, d'étudiants, d'artistes et de professionnels comptaient sur le sens d'appartenance des Québécois pour faire triompher leur option. Dans ce genre de méthode, le support des média est important. La propagande du oui faisait appel à la fierté nationale.

Au fond, les deux camps ne visaient tout simplement pas les mêmes clientèles. La propagande du non cherchait à toucher des groupes particuliers aux intérêts précis. Le raisonnement fut juste. On peut donc dire que les méthodes préconisées par le comité du non étalent plus

3 - Maurice Lamontagne, "Fédéralisme ou association d'états indépendants", Etudes internationales, v. 8, no. 2, juin 1977, p. 225 
adaptées à notre société industrielle. Les hommes d'affaires ont appuyé en majorité le camp du non. La Chambre de commerce du Québec l'a fait aussi.

Au niveau de la presse québécolse, on a assisté à des prises de position de part et d'autre. Par exemple, le quotidien La Presse était pro-fédéraliste avant même le débat. La presse régionale n'a pas pris position sur la question débattue lors du référendum. On peut même dire que les éditoriaux de la presse québécolse ont penché en faveur du non.

De plus, 11 existait des différences marquantes au niveau de l'Interprétation et de la couverture des événements entre la presse francophone et anglophone. La presse francophone se concentralt sur la couverture des protagonistes officiels. La presse anglophone donnait en plus son opinton. "Ainsi, on écrit qu'aucune loi en Ontario n'empêche une compagnie de fonctionner en français, alors qu'on ajoute à une autre occasion que l'Ontario possède un excellent record en ce qui a trait aux écoles françaises". (4) Au fond, la presse francophone a couvert le référendum comme une élection ordinaire.

4 - Yves Gagnon, "Les quotidiens québécols et le référendum", Communication et information, v. 3, 1980, p. 174 
De plus, 11 y a certains groupes qui n'ont pas participé au débat: 1'extrême gauche. "Pour ce falre, ils ont voulu donner la parole a des gens de gauche, de cette gauche qui a certes été exclue de 1a campagne référendaire par des règles de jeu contraignantes qui ob1igeaient les participants à se regrouper sous des comités-parapluies, mais qui s'est aussi exclue par l'ambiguité de son message et de sa position". (5) Socialisme et indépendance ne vont plus nécessairement de pair.

Lors du référendum de 1980 , les Québécois devalent choisir entre la souveraineté-association du P.Q. et le fédéralisme canadien. Ils ont rendu leur verdict au mois de mai. Ce fut une date mémorable au niveau de 1a participation populaire. En effet, elle fut de $87.3 \%$. C'est un nouveau record. Les taux de participation ont été donc hauts de part et d'autre.

Chez les francophones, le non a remporté aussi la victoire. La marge fut extrêmement mince cependant. "A ce jour, nul ne sait si nos

5 - Nicole Laurin-Frenette et Jean-François Léonard, "L'Impasse, enjeux et perspectives de 1'après référendum", Recherches sociographiques, v. 22 , no. 3 , sept.-déc. 1981 , p. 419 
deux cinquièmes de Oui représentèrent ce soîr-1a $49 \%$ ou $51 \%$ du Québec français". (6) Il y a eu de plus un clivage au niveau des âges. Les jeunes semblaient plus favorables à la souveralneté-association que les personnes plus agées. C'est même un fait qui fut assez juste pour 1'ensemble des francophones. Les autres facteurs ont eu donc peu d'influence dans le cholx du vote: revenu, occupation, appartenance syndicale, éducation et même le sexe. Au fond, $11 \mathrm{n}^{\prime} \mathrm{y}$ a pas eu de différence significative entre le comportement des hommes et celui des femmes. Les femmes n'ont pas eu en général un comportement réellement spécifique lors du référendum de 1980 .

Les journaux ont réag1 différemment aux résultats du référendum. Ce furent les journaux anglophones qui ont êté le plus loln dans leur analyse du vote. En particulier The Gazette, qui fut probablement le quotidien le plus radical de tous. Il écrit que "c'est la fín du rêve pour les indépendantistes". Quant aux journaux francophones (Le Devoir,

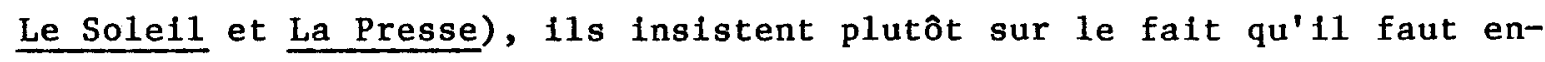
treprendre des réformes constitutionnelles pour donner sa juste part au Québec dans les domaines qui luf reviennent.

6 - René Lévesque, Attendez que je me rappe11e..., Montréa1, Québec/Amérique, $1986, \mathrm{p} \cdot 414$ 
Analyse de contenu des journaux locaux

Le référendum de 1980 fut une autre consultation publique majeure. Le mols de mal fut la période culminante où s'est joué le résultat de cette consultation. La presse saguenéenne a décrit cet événement de quelle façon? C'est dans les médla écrits que nous trouverons des éléments de réponse. Les éditoriaux du Quotidien de Chicoutimi sont restés neutres. Le Progrès-Dimanche et 1'Étoile du Lac ont-ils subi le même phénomène? Est-ce qu'on s'est concentré plutôt sur la couverture des protagonistes officiels? Volla les questions que nous nous posons.

Le référendum n'était pas un événement politique "ordinaire". Les média francophones en général 1'ont couvert ainsi. Le Progrès-Dimanche et L'Étolle du Lac ont-11s suivi la règle? Ou ont-11s été 1'exception? De part et d'autre les hommes politiques ont eu un rôle important a jouer. Nous verrons leurs divers arguments pour le oul ou pour le non. Les deux journaux analysés sont des hebdomadaires. L'un couvrait le Saguenay (Le Progrès-Dimanche) et l'autre le Lac Salnt-Jean (L'Étolle du Lac). On aura donc une vue d'ensemble de l'information au Saguenay-Lac Saint-Jean. C'est un facteur à ne pas dédaigner. Le Progrès-Dimanche a cette époque a souffert de "troubles syndicaux". Cependant, c'est seulement à une occasion qu'il ne sera pas imprimé normalement. 


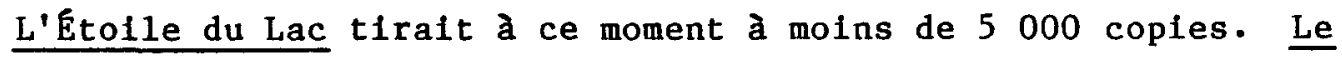
Progrès-Dimanche dépassait ce dernier. I1 était largement dans la catégorle des hebdos français de 5000 exemplaires et plus. Notre région est définitivement dans l'ère moderne. La télévision et la radio sont le lot de tous les foyers saguenéens. Le Progrès-Dimanche est un solide journal d'Information régtonale et nationale. L'Étolle du Lac parle aussi souvent de politique que d'agriculture. Le contenu "politique" est presque aussi important que celul du Progrès-Dimanche. C'est un falt nouveau. Dans l'échantillon analysé, on retrouve des éditoriaux, des lettres de lecteurs, des résumés de réunions politiques, des condensés de presse étrangère, des prises de position, des débats économiques et des communications des comités du oui et du non.

Notre échantillon rassemble 69 articles. I1 est aussi imposant que celui du chapitre précédent. Il est nettement plus diversifié à cause, par exemple, de son contenu économique. Il est aussi plus gros en terme de volume. L'échantilion pris est 1a totalité des documents du

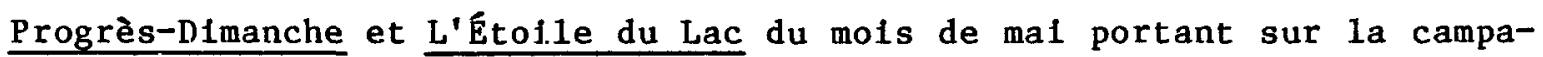
gne du référendum de 1980 et les résultats de cette consultation.

La méthodologie est la même que dans les chapitres antérieurs. 
C'est une analyse dirigée dans le sens que nous tentons de vérifier ou d'infirmer une hypothèse: dans un premler temps les journaux analysés ont couvert le référendum comme une élection ordinafre, et dans un deuxième temps, les messages des comités du oul et du non ont eu une place égale dans les média réglonaux. Notre étude porte sur 69 articles (38 du Progrès-Dimanche et 31 de L'Étolle du Lac). Notre traval1 couvre une période d'un mois (mai). C'est pendant cette période que le débat politique était à son comble et que l'émotion, à la suite de cet événement, fut vive. Dans les prochaines pages, les tableaux représentant les catégorles retenues ainsi que leur fréquence par journal suivent. 
TABLEAU I

CATEGORIES

ARGUMBTSS POUR LE OUI

ARGUMENTS POUR LE NON

ARGUMRNTS CONTRB LE OUI

ARGUMENTS CONTRE LE NON

IDENTITE DES ACTEURS 
95 Arguments pour le oui

13 Arguments pour le non

23 Arguments contre le oul

10 Arguments contre 1e non

291 Identité des acteurs

66 Arguments pour le oui

18 Arguments pour le non

21 Arguments contre le oui

4 Arguments contre le non

92 Identité des acteurs 
TABLEAU III

ARGUMENTS POUR LE OUI

(FORHULES LES PLUS FRAPPANTES)

MOTS

FREQUENCES

Aspiration juste et légitime 1

Avoir une porte sur le marché 1

Auto-détermination 1

Cesser de se faire gouverner par les autres 1

Chance de percer sur le plan international 1

Cause emballante 1

Débloquer 1'Imbrog1io constitutionnel 2

Égal à égal 11

Égalité des deux peuples 1

Entre deux inconnus on choisit le sien 1

Épanouissement légitime 1

Fédéralisme renouvelé impossible dans les faits 1

Faire nous-mêmes les lois 1

Halne des anglais $\quad 2$

La nature même d'un peuple 1

Les anglais devront négocier 7

Maturité politique 1

Mémoire 1

Négocier en position de force, une nouvelle entente,

le changement 3

Nouvelle vie 3 


\section{TABLEAU III}

\section{ARGUMENTS POUR LE OUI}

\section{(SUITE)}

Permet aux québécois d'accroftre le pouvoir de leur gouvernement

Pour un déblocage constitutionnel

Représente un nouveau départ

Sulvre le cours de 1'histolre, de notre histoire

Se prendre en main

Solidaires

Se doter d'un état plus puissant

Temps de s'affirmer

Trudeau fait le contraire de ce qu'il dit

Trouver une nouve1le entente 


\section{TABLEAU IV}

\section{ARGUMENTS POUR LE NON}

\section{(FORMULES LES PLUS FRAPPANTES)}

MOTS

FRÉQUENCES

Amener la continuité du pays

A 1'aventure que vous propose le parti Québecols

Gardons accès aux services du ministère fédéral de la santé

L'indépendance n'est pas nécessałre

Mon nom est québécois

Mourir en Canada

Pas prête à faire l'indépendance

Pas d'avenir à se rapetisser

Autres 
TABLEAU V

ARGUMENTS CONTRE LE OUI

(FORHULES LES PLUS FRAPPANTES)

MOTS

FRÉQUENCES

Appel au sens de la responsabilité 1

État ethnique 1

I1 faut 1ire la stratégie de Doris Lussier 1

Le véritable argument du gouvernement actuel est de faire

1 'indépendance

La fin, 1a raison d'être et 1 a raison d'agir du parti

Québecois, c'est la souveralneté du Québec et 1'éta-

blissement d'un rêgime social-démocrate

La population n'est pas prête à faire 1'indépendance 1

On ne sait pas ce qu' 11 y a dessous 1

Régime totalitaire qui se dessine 1

Soyez sérleux 1

$\begin{array}{ll}\text { Tribalisme } & 1\end{array}$

"Tous les moyens sont bons pour gagner une
élection..." (Doris Lussier)

Une façon vulgaire de mousser son produft 1

Une route difficile, parsemée d'embûches et conduisant

à 1a séparation définitive du Québec du Canada 1

Une personne intelligente ne peut dire oui 1

$\begin{array}{ll}\text { Autres } & 30\end{array}$

TOTAL 
TABLEAU VI

ARGUMENTS CONTRE LE MON

\section{(FORMULES LES PLUS FRAPPANTES)}

MOTS

FRÉQUENCES

Aliénation 1

$\begin{array}{ll}\text { Dépendance } & 1\end{array}$

Inacceptable $\quad 1$

Impossible dans les faits 1

Le document de M. Trudeau est catastrophique 1

La dictature de M. Trudeau 1

La tour de Pise penche toujours du même côté 1

On n'accepte pas ou n'approuve pas les conclusions

du Livre belge $\quad 2$

Pure Fantaisie 1

Va-t-on cesser de se moquer des québécols 1

Voter NON, c'est accepter de perdre beaucoup

de pouvoirs 1

Quand je lis 1'histoire, je ne peux pas aimer

les anglais 1

Une voie sans issue 1

$\begin{array}{ll}\text { TOTAL } & 14\end{array}$ 
Artistes: (Colette Boky, Gaston Miron, Doris Lussier) 5

Avocat $\quad 5$

Commis 3

Échevin 1

$\begin{array}{ll}\text { Enseignant } & 1\end{array}$

$\begin{array}{ll}\text { Etudiant } & 10\end{array}$

Économistes: (Serge Moussaly, Paul Prévost) 10

$\begin{array}{ll}\text { Femmes au foyer } & 10\end{array}$

Hommes de la rue 20

Médecın $\quad \cdot \quad 1$

$\begin{array}{ll}\text { Malres } & 10\end{array}$

Politiciens provinclaux: (Ryan, Lévesque, Parizeau,
Lazure, etc.)

Politiciens régionaux: (Marc-André Bédard, Marcel 100

$\begin{array}{ll}\text { Autres } & 7\end{array}$

TOTAL 
Résultats de 1'analyse de contenu

A la lumière de cette enquête, on peut affirmer que la presse réglonale a couvert le référendum de 1980 comme "une élection ordinaire". Les éditorialistes ont toujours solgneusement évité de prendre position. On tente de rester résolument hors du débat référendaire. Dans ce rôle d'arbitre, les journaux régionaux ont largement réussi leur mission d'information vis-à-vis les deux options. On laisse parler les deux camps librement. Marcel Lessard et Marc-André Bédard ont par exemple tous les deux leur tribune.

La catégorie "arguments pour 1e ouf" obtient un total de 161 fréquences. Si on lui ajoute la catégorie "arguments contre le non" on arrivera au total de 175 fréquences. On expliquera cette supériorité notolre de 1'option du ouf sur le non dans les journaux analysés par le falt que les tenants du non n'expliquent pas réellement les raisons de leur refus. Ils disent simplement qu'lls sont pour le non sans plus développer. Certains vont jusqu'à dire que le référendum est une perte de temps. Le camp du oul est nettement plus agressif. L'action des regroupements pour le oui en est une preuve frappante. Des dizaines de personnes expliquent les raisons de leur adhésion à longueur de page. 
La seule prise de position que prennent les éditorialistes est de déplorer la tendance que prend le débat référendaire. Alnsi 1'éditoriallste de L'Étoile du Lac, Robert Paradis, titre: "Une triste campagne référendaire". L'extrémisme est condamné. La catégorie "identité des acteurs" obtlent une fréquence de 383. C'est la plus importante catégorie. On retrouve tous les intervenants possible de la société québécoise: poètes, artistes, politiciens provinclaux et fédéraux, économistes, élus municipaux, etc.

On fait aussi abondamment référence aux déclarations de l'homme de 1a rue. C'est un faft nouveau, car dans les chapitres précédents on n'affectionnait guère ce genre de procédé. On volt donc qu'on ne 1'a pas tout à fait couvert comme une "élection ordinaire". Un autre fait sortant de 1'ordinalre: on donne une place importante aux avis des ainés. On leur consacre des pages entières.

Le nom qui revient le plus souvent dans la catégorie "identité des acteurs" est le nom de Claude Ryan, mais c'est plutôt pour le désavouer $q u^{\prime}$ on en fait mention. En effet, lorsque $M$. Ryan a dit que si notre région a voté massivement pour le oul, c'est parce qu'elle n'est 
pas gâtée au niveau des communications; il ne s'est pas falt beaucoup d'amis dans la presse écrite. L'éditorialiste du Progrès-Dimanche, Denis Tremblay, titre d'ailleurs: "Ryan-la Gaffe".

Le monde religieux n'a guère une place très marquée dans ce débat. En effet, on n'est plus au temps où l'autorité religieuse dictait la conduite à suivre au peuple laïque. Le clergé évite même de prendre position officiellement. Seuls les Pères Capucins mentionnent qu'ils voteront pour le oui. On falt une rectification pour dire que c'est seulement une petite minorité quí se prononcent ouvertement pour le ouí, les autres gardant résolument le secret sur le vote. Dans les comités certains prêtres se déclareront individuellement en faveur du oui. Les hautes autorités de 1'Église catholique resteront hors du conflit. On invite plutôt les chrétiens à réfléchir sur leurs responsabilités dans la société.

On assiste à un débat entre économistes spécialisés dans la région du Saguenay-Lac Saint-Jean: M. Serge Moussaly et M. Paul Prévost. Monsieur Moussaly ne votera pas pour 1'une ou 1'autre des options mais 11 concède de grandes qualités au fédéralisme canadien. Monsieur Prévost considère qu' 11 est temps de s'affirmer en tant qu'État moderne en disant oui au référendum. 
La catégorie "arguments contre le oui" obtient un total de 44 fréquences. Le fait intéressant dans ce genre de catégorie, c'est de voir de quelle manière on va contester la thèse de l'adversaire. Par exemple, le camp du non utilise beaucoup les écrits de Doris Lussier pour "démolir" la souveraineté-association. On accuse ainsi d'intentions malhonnêtes le Parti québecois et ses partisans. On fait aussi référence à un régime totalitalre qui se dessinerait après le oui. Une supposition qui est peu rassurante pour l'électeur moyen.

La catégorie, "arguments contre 1e non" n'obtient que 14 fréquences. C'est peu. Certains mots font évidemment surface: aliénation, dépendance inacceptable, etc. On semble moins agressif dans la dénonciation du système fédéral. Les mots les plus durs viennent de particuliers qui écrivent au journal. Par exemple, l'un d'eux écrit: La dictature de M. Trudeau". L'argument qui revient le plus souvent c'est qu'on n'est pas satisfait du livre beige de M. Ryan. On peut donc affirmer que lors du débat référendaire, le ton des discours dans la presse régionale est resté dans des normes "très civilisées". Les média écrits de notre région ne furent pas les plus radicaux. On ne parle pas de guerre civile "verbale". 
Si on fait une récapitulation générale au niveau de la signification du message analysé, on arrivera aux conclusions suivantes: tout d'abord le camp du oul a mené une campagne plus agressive et a mieux développé ses arguments dans la presse réglonale. Le camp du non a été sur la défensive. Finalement, si on fait un résumé au niveau des fréquences, on arrive à ces résultats: 1a catégorie "arguments pour le oui" obtient le nombre de fréquences le deuxième plus élevé solt 161. La catégorie "arguments pour le non" arrive en quatrième place avec 31. La catégorie "arguments contre le oul" arrive en troisième place avec 44. La catégorle "arguments contre le non" arrive en clnquième avec 14. La première place est tenue par 1a catégorie "identité des acteurs" avec 383 . Un dernier tableau illustre la situation. 


\section{CLASSEMEIT PAR CATEGGORIE}

1. Identité des acteurs

2. Arguments pour le oul

3. Arguments contre le oui

4. Arguments pour le non

5. Arguments contre le non 
CONCLUSION

La première conclusion qui s'impose, veut que les média écrits du Saguenay ont été continuellement hantés par les conflits politiques qui ont secoué dans ce siècle le Canada et le Québec. Les média écrits du Saguenay Lac Saint-Jean n'ont jamais été des espaces fermés. Le décalage au niveau des grands débats polltiques canadiens n'existe pas. La presse écrite régionale n'est pas exempte des consultations publiques qui ont fait la manchette au Québec. Il n'y a pas eu de pénurie d'information écrite. Le domalne des mass médla écrites ne souffre pas d'une "rupture" avec le reste du pays. La population du Saguenay ne fut pas laissée dans 1'ignorance. Cette constatation ne peut être mise en question. Notre travail le prouve raisonnablement. Le doute n'a plus sa place désormais. Notre région a suivi l'évolution du reste du Québec. La dynamique historique est la même dans les deux cas. Cette situation est déjà une preuve en soi.

A titre d'exemple, le rôle du clergé a changé fondamentalement. Si on regarde dans les grandes 11gnes, notre travail, on notera un déclin sensible et visible. En 1942, alors que 1'influence de 1'Église cathol1que étalt à son sommet, le clergé parle de concert avec le consell central des Syndicats nationaux catholiques du diocèse de Chicoutimi. Cependant, 11 ne faut pas s'étonner de cette situation puisque le premier syndicat catholique en Amérique du Nord a été fondé à Chicoutimi au début de ce siècle. 
On ne retrouvera plus une telle manifestation dorénavant. En 1970, évidemment le clergé condamnera durement l'exécution de Plerre Laporte. Il n'est plus auss 1 puissant que le pouvoir civil cependant. L'autorité du clergé est désormais spirituelle et morale. Son pouvolr s'arrête 1à. Finalement, en 1980 , le clergé refuse tout simplement de prendre position officlellement dans les média écrits. La révolution tranquille a changé bien des choses et cela même à un niveau régional.

Dans le Québec moderne, ce sont les polfticlens et les autorités civiles qui accaparent 1'attention des média écrits. Ils détiennent le monopole de 1a parole écrite. Cette situation se reflète au SaguenayLac Saint-Jean. Notre région a été toujours un bastion du fait français en Amérique. Ses prises de position par rapport au Canada anglais en sont une preuve frappante. Par exemple, lors des débats publiques de 1917 et 1942, les profondes contradictions qui existaient entre le Canada anglais et le Canada français se retrouvent précisément dans la presse du Saguenay. L'écrasante majorité du falt français au Saguenay-Lac SaintJean semble en être 1a cause. Les deux votes lors de ces consultations publiques rejoignent les prises de position des autres communautés francophones hors du Québec. Le facteur ethnique pourrait expliquer cette situation. Son élolgnement géographique des grands centres aurait renforcé ce dernier fait. Si le cardinal Villeneuve, archevêque de Québec, a pris parti pour la politique de guerre du gouvernement King, il semble- 
rait que son message a eu peu de répercussion dans les média écrits du Saguenay. On peut même dire que le bas clergé était plus sympathique à 1a cause des anti-conscriptionnistes. L'action du conseil. central des Syndicats nationaux catholiques du diocèse de Chicoutimi en 1942 en est un fait assez visible.

Le meurtre de Pierre Laporte fut condamné par les journaux francophones du Québec. On retrouve cette condamnation unanime dans les média régtonaux pendant la crise d'octobre. Encore une fois, le Saguenay-Lac Saint-Jean n'est pas en marge du reste du Quêbec. Les journaux régionaux ont donc eu des positions comparables à celles adoptées par les imprimés de Québec et de Montréal.

Les francophones du Saguenay-Lac Saint-Jean ont voté en majorité pour le oul. C'est d'aflleurs un des endrolts où $i 1$ y en a eu le plus et où la stratégie du Parti québécols a mieux rejoint les masses. Son élolgnement des grands centres urbains n'a jamais été la cause de son nationalisme profond. C'est plutot le facteur ethnique qui serait la cause principale de ce fait. De plus, le petit nombre d'anglophones qui réside au Saguenay-Lac Saint-Jean a aussi beaucoup compté. La communauté anglaise n'a jamais été très nombreuse dans notre région. On peut même 
dire qu'elle s'est "assoclée" à la longue au falt françals dans notre réglon. La réalité des autres grands centres urbains du Québec est différente fondamentalement.

Notre hypothèse s'est vérifiée: Une région comme la nôtre même si elle est éloignée des grands centres n'a jamais été un espace clos par rapport au reste de la société québécoise. Les média écrits du Saguenay ont été touchés par les grands débats politiques qui ont secoué le Québec et le Canada. On retrouve dans notre région les profondes contradictions canadiennes qui bouleversent par vague notre pays. En effet, ce sont les mêmes êlites qui prennent la parole dans les journaux régionaux. Par exemple, lors des émeutes de 1918 , ce sont les autorités civiles et militaires qui commenteront "l'événement", l'Église catholique se gardant bien de se mêler de l'affaire. Mals lors de la deuxième conscription, on aura des sympathies visibles pour les anti-conscriptionnistes régionaux. Cette attitude transparaft largement d'ailleurs dans la presse de 1'époque.

Lors de la crise d'Octobre 1970, 1'Église catholique régionale a dénoncé vigoureusement les actes de violence (en particuller le meurtre de Plerre Laporte). La population saguenéenne partageait en majorité 
cette position. En 1980 , le clergé s'est abstenu d'intervenir dans le débat polftique, une position d'allleurs qui ne se différenciait pas des journaux francophones de l'époque. Certains prêtres ont pris cependant parti Individuellement pour l'une ou l'autre option. L'évolution sociale et politique du Saguenay-Lac Saint-Jean est sensiblement la même qu'a subie l'ensemble de la société québécoise. Au fond une région comme la nôtre n'est qu'une extension d'un tout plus vaste, solt le Québec. Dire que les cholx politiques du Saguenay-Lac Salnt-Jean sont dictés par un "élolgnement" des grands centres urbalns n'est tout simplement pas vral.

Au contralre, quand on examine solgneusement ses choix politiques, 11 apparait une cohérence qui était inconnue jusqu'à maintenant avec le reste du fait français en Amérique du Nord. Consciemment ou inconsclemment, notre réglon s'est toujours rangée lors des grands débats politiques canadiens analysés, derrière la majorité francophone du Québec, mals minorité dans l'ensemble géographique canadien.

Les conflits profonds qui hantaient le Canada et le Québec se sont répercutés au Saguenay-Lac Salnt-Jean. La crise part toujours du 
centre pour se communiquer à sa périphérie. Ce n'est pas le contraire qui se passe généralement. L'ensemble à part n'existe plus. Quand Claude Ryan a déclaré pour expliquer en 1980 la défaite de son comité au Saguenay-Lac Saint-Jean, que cet état de choses était imputable au mauvais état des communications, 11 a falt une erreur d'appréciation. La réaction des média écrits et des gens en est une preuve. E1le fut immédiate et violente: certains journalistes $1^{\prime}$ ont même qualifié du surnom de Ryan-1a-Gaffe et plusieurs de ses partisans l'ont désavoué publiquement dans des lettres. C'est le fait francophone plus important qu'alleurs qui est le grand responsable de ces choix contestés. Pourtant en 1976, lors du raz de marée péquiste, personne ne 1'a falt. C'est peut-être que le Saguenay-Lac Saint-Jean est tout simplement plus conséquent avec luimême.

Le Saguenay-Lac Saint-Jean a une des plus grandes majorités de francophones au Québec. C'est un fait que tout bon politicien (fédéral ou provincial) dolt prendre en considération dans une campagne électorale. Notre région a toujours été un fief du nationalisme québécois. L'élément culturel français pèse donc beaucoup dans la balance. La politique des journaux réglonaux ne se dissocle pas réellement des imprimés "nationaux". Cependant on peut être fier de 1a manière dont ils ont traité les événements analysés. 
Par exemple, lors des émeutes de Québec de 1918, 1es communiqués de la presse régionale au sujet de ce drame n'ont souffert d'aucune "exagération" notoire. Même chose pour 1'élection de 1942 où se jouait le sort de la conscription. Quant au meurtre de Pierre Laporte en 1970, on peut dire que la presse régionale a respecté le ton tragique que prenalt cet événement sans trop en mettre. Finalement en 1980, lors du référendum, les média écrits ont couvert cette consultation comme une élection ordinaire (même si c'était peut-être le débat politique le plus important qu'alt connu notre pays). Une distance réelle quil falt plutôt honneur a des média qu'on qualifle quelquefols de locaux alors que, dans les grands centres urbalns, les traitements de l'information ont pris une tournure tout autre.

Les espaces locaux, provinclaux et nationaux sont un tout intimement lié au Canada. Par exemple, les grands conflits politiques nationaux (référendum, constitution, 1ibre-échange, etc.) se répercutent jusqu'aux régions. Est-ce que cela se prodult dans tous les systèmes fédéralistes du monde? Est-ce que seulement de telles choses se produisent aux Etats-Unis qui sont d'allleurs nos plus proches voisins? A 1'origine le Canada fut fondé sur une dualité: le fait français et le fait anglais en Amérique du Nord. Les premières lois de ce pays ont été d'inspiration anglaise. Les bases du fédéralisme canadien l'ont été aussi. Pourtant ce sont deux peuples différents par leur langue et leur religion qui 
l'ont engendré. Ou plutôt lis se le sont partagés. Les Amérindiens $n^{\prime}$ ont pas eu le droit à la parole lors de la fondation du Canada en tant qu'État-nation. Au fond, à la lumière de ces derniers falts, 11 ne faudrait donc pas s'étonner qu'il soit secoué périodiquement par des crises internes. Le Canada porte ses contradictions en 1 ui, pour 1 e mellleur ou pour le pire.

Ce n'est plus de l'équilibre, mals du trapèze que falt le gouvernement fédéral par moment. Pourtant, c'est son ldentité même par rapport à son voisin américain qui est en jeu. Évidemment, il y a beaucoup d'autres communautés francophones en Amérique du Nord. Un recensement de 1970 Indiquait que seulement en Nouvelle-Angleterre, un million de francophones vivent en français au domicile. Ils disposent même d'organes de presse. I1 y a $30 \%$ de francophones dans le New-Hampshire, $10 \%$ dans 1e Maine et autant dans le Massachusetts. En Loulsiane, un demi-million de personnes se disent francophones et vivent en français. On sait aussi que 200000 québécols vivent en permanence en Floride, soit presque la population du Saguenay-Lac SaInt-Jean dans ce seul état américain. En conclusion, le Québec reste encore la plus importante communauté francophone en Amérique du Nord mais avec une diaspora diversifiée.

Les structures fédératives sont par définition des situations 
conflictuelles. Le consensus défini par la loi fondamentale et explicité par le système juridique et politique est toujours remis en question partlellement en fonction des intérêts particuliers des éléments composants des fédérations. Seule une ldéologie commune peut rapprocher les participants. La question est de savoir si ces conflits politiques sont constructeurs ou destructeurs de 1'union fédérale canadienne. En 1980, 1e Québec a dit non à la souveraineté-association du Parti québécols. Il a dit donc oui au fédéralisme canadien. Est-ce que le reste du Canada dira oui a son tour au Québec dans le futur?

Au fond si le Québec étalt assimilé culturellement au reste du monde anglophone en Amérique du Nord, on peut douter fortement que le Canada garderait son identité en tant qu'entité culturelle et politique indépendante de son grand voisin américain. Le fédéralisme canadien est tirallé par ses profondes tensions régionales. Les particullarismes de notre pays s'expriment également en Ontario et en Alberta. Les procha1nes difficultés que devra affronter le Canada pourraient blen venir d'allleurs que du Québec. C'est pour cela que ce genre de travall a une Importance grandissante pour $1^{\prime}$ avenir de ce pays. C'est donc dire que c'est un nouvel univers qui s'ouvre aux yeux de l'historien et du chercheur en général. A partir d'une région, on peut déboucher sur des perspectives qui touchent une nation. Les études régionales sont une discipline très nouvelle et ses possibilités paralssent prometteuses pour le moins. 


\section{BIBLTOGRAPHIB}

I. OUVRAGES GENERAUX

Sur la conscription de 1917:

\section{LIVRES}

Armstrong, Elizabeth Howard, The Crisis of Quebec, 1914-1918: with and introd. by Joseph Levitt, Toronto: Mc Clelland and Stewart, 1974, 275 p.

Bernard, Julienne, Mémolres chapais, Montréal: Fides, 1961. 3 volumes

Bélanger, Réa1, L'impossible défi: Albert Sevisny et les conservateurs fédéraux: (1902-1918), Québec: Presses de I'Université Lava1, 1983 . 368 p.: 111 .

Bélanger, Réal, Paul-Émile Lamarche: le pays avant le parti: (1904-1918), Salnte-Foy: Presses de 1'Université Laval, 1984. 439 p.

Bêlanger, Réal, Wilfrid Laurier : quand la polltique devient passion, Réal Bêlanger Québec : Presses de 1'Unfversité Laval; Montréal: Entreprises Radio-Canada, 1986. 484 p.: $111 .$, portr.

Bouchard, Télesphore Damlen, Mémolres de T.D. Bouchard / préface du Réverend Père Albert Mllot, O.P Montréal: Beauchemin, 1960. 3 volumes

Choquette, Robert, Langue et religion: histoire des conflits anglo-français en Ontarto, 2e éd. Ottawa: Éditions de l'Université d'ottawa, 1980.268 pages

Filteau, Gérard, Le Québec, le Canada et 1a guerre 1914-1918, Montréal: Editions de 1'aurore, 1977,231 pages

Granatstein, J.L. et Hitsman, J.M., Broken Promises: A History of Conscription in Canada, Toronto: Oxford University Press, 1977, 281 pages

Jacques, Michel, La participation des canadiens-français à la guerre, Montréal: Éditions de 1'A.C.F., 1938, $188 \mathrm{p}$.

Pinsonneault, Gérard, La propagande de recrutement militaíre au Canada: 1914-1917: Essal en histoire des mentalités, Sherbrooke, Les Ếdtions de 1'Université de Sherbrooke, 1984, 200 p.

Provencher, Jean, Québec sous la lo1 des mesures de guerre, 1918, pref. de Fernand Dumont, Montréal: Éditions du Boréal Express, 1971, 146 p.

Wade, Mason, Les canadiens français de 1760 a nos jours: trad. de 1'anglais par Adrien Venne avec le concours de Francis Dufau-1abeyrie, Ottawa: Cercle du Livre de France, 1963. 2 volumes 


\section{PERIODIQUES}

Le Progrès-Dimanche: avril à mai 1918, avril à mal 1942, octobre 1970, mai 1980

L'Étolle du Lac: avril à mai 1918, avril à mai 1942, 1964, octobre 1970, mal 1980

Sur la conscription de 1942:

1. LIVRES

Barrette, Antonio, Mémoires, Montréal: Beauchemin, 1966, 300 p.

Black, Conrad, Duplessis, Montrêal: Les Éditions de 1'homme, 1977, 2 volumes

Chaloult, René, Mémoires politiques, Montréal: Éditions du Jour, 1969. $295 \mathrm{p}$.

Granatstein, J.L, Canada's War The politics of the Mackenzie King Governement, 1939-1945, Toronto: 0xford University Press, 1975. 436 pages

Groulx, Lionel Mes mémolres, Montréa1: Fides, 1970, 4 volumes

Laurendeau, Andrê, La crise de la conscription, 1942, Montréal: Éditions du jour, $1962,157 \mathrm{p}$.

Pickersg111, John Whitney et Forster, D.F., The Mackenzie King Record, Toronto: University of Toronto Press, 1968,2 volumes

Quinn, Herbert F. The Union nationale: Quebec Nationalism from Duplessis to Levesque, Toronto: UnIversity of Toronto Press, 1979. 342 p.

Rumi11y, Robert, Maurlce Duplessis et son temps, Montréal: Fides, 1973, 2 volumes

PERIODIQUES

Granatstein, J.L., "Le Québec et le pléblscite de 1942 sur la conscription", Revue d'histoire de 1'Amérique française, vol. 27 , no 1 (juin 1973): $43-62$.

Sur 1a crise d'Octobre:

LIVRES

Dumont, Fexnand, La vigile du Québec: octobre 1970 - 1'1mpasse, Montréal: Hurtubise HMH, 1971. 234 p. 
Fournier, Louis, F.L.Q.: Histolre d'un mouvement clandestin, Montréa1: Québec/Amérique, 1982, 509 p.

Laurendeau, Marc, Les Québécols violents: un ouvrage sur les causes et 1a rentabilité de la violence d'inspiration politique au québec, Montréal: Editions du Boréal Express, 1974, 240 p.

Morf, Gustave, Le terrorisme québécols, Montréal: Editions de 1'homme, 1970,219 p.

Pelletier, Gérard, La crise d'Octobre, Montréal: Éditions du jour, 1971, 265 p.

Québec (Prov.), Commission d'enquête sur des opérations policières en territoire québécols, Keable, Jean, T. Press, Québec: Ministère de la Justice, 1981,451 p.

2. PERIODIQUES

La Presse: 1964

Le Devoir: 1964

Le Sole11: 1964, octobre 1970

Parti pris: $1963-1968$

Sur le référendum

LIVRES

Bergeron, Gérard, Ce jour-1a... le référendum, Montréal: Quinze, 1978, $265 \mathrm{p}$.

Bol1y, Robert et collaborateurs, Québec: un pays incertain, réflexion sur 1e Québec post-référendaîre, Montréal: Québec/Amérique, 1980, $312 \mathrm{p}$.

Lévesque, René, Attendez que je me rappelle..., Montréal: Québec/Amérique, $1986.525 \mathrm{p}$.

PERIODIQUES

Gagnon, Yves, "Les quotidiens québécols et le référendum", Communtcation et Information, vo1. 3, 1980, pp 170-181.

Lamontagne, Maurice, "Fédéralisme ou association d'états indépendants", Etudes Internationales, vo1. 8, no 2 (juin 1977), pp 208-230. 
Laurin-Frenette, Nicole et Léonard, Jean-François, "L'impasse, enjeux et perspectives de 1'après référendum" Recherches sociographiques, vo1. 22, no 3 (sept.-déc. 1981), p. 419-420.

B. SPÉCIALES

Sur la région du Saguenay-Lac St-Jean

LIVRES

Blanchard, Raoul, L'Est du Canada français "province de Québec", Paris: Librairie Masson: Cie 8, 1935, 2 volumes

Boudreault, Pierre W., Luttes réglonalitaires et société post-industrielle, Dix ans d'actions soclopolitiques au Saguenay, Sagamie/Québec, $1986185 \mathrm{p}$.

Lapointe, Raoul, Histolre de 1'Imprimerie au Saguenay (1879-1969), Chicoutimi: La société historique du Saguenay, 1969, 291 p.

Etudes sociologiques

1. LIVRES

Burdeau, Georges, Traité de sclence politique, Tome VIII: La démocratie et les contraintes du nouvel age, Paris: L.G.D.J. $2 \overline{0 \text { et } 24 \text {, rue }}$ Soufflot, 1974, 673 p.

Burdeau, Georges, Traité de science politique, Tome X: La rébellion du social, $1977,391 \mathrm{p}$.

Dumont, Fernand, Le lien de 1'homme: La culture comme distance et mémo1re, Montréal: H.M.H., 1971, 233 p.

Gauthier, Benoît, Recherche sociale: De 1a problématique à la collecte des données, Québec: Presses de 1'Université du Québec, 1984, 504 p.

Grawitz, Madeleine, Méthodes des sciences sociales, Paris: Dallonz, 1979, 1102 p.

Mandras, Henri, Eléments de sociologie, Paris: A. Co11in, 1967, 249 p. 